\title{
THE VORONOI TESSELLATION CLUSTER FINDER IN 2+1 DIMENSIONS
}

\author{
Marcelle Soares-Santos ${ }^{1,2}$, Reinaldo R. De Carvalho ${ }^{3}$, James Annis ${ }^{1}$, Roy R. Gal ${ }^{4}$, Francesco La Barbera ${ }^{5}$, \\ Paulo A. A. Lopes $^{6}$, Risa H. Wechsler ${ }^{7}$, Michael T. Busha ${ }^{7}$, and Brian F. Gerke ${ }^{7}$ \\ ${ }^{1}$ Fermi National Accelerator Laboratory, Batavia, IL, USA; marcelle@ fnal. gov \\ 2 Instituto de Astronomia, Geofísica e Ciências Atmosféricas, Universidade de São Paulo, São Paulo SP, Brazil \\ ${ }^{3}$ Divisão de Astrofísica, Instituto Nacional de Pesquisas Espaciais, São José dos Campos SP, Brazil \\ ${ }^{4}$ Institute for Astronomy, University of Hawaii, Honolulu, HI, USA \\ 5 INAF-Osservatorio Astronomico di Capodimonte, Salita Moiariello 16, 80131 Napoli, Italy \\ ${ }^{6}$ Observatório do Valongo, Rio de Janeiro RJ, Brazil \\ ${ }^{7}$ Kavli Institute for Particle Astrophysics and Cosmology, SLAC National Accelerator Laboratory, Stanford University, Stanford, CA, USA \\ Received 2010 August 2; accepted 2010 November 13; published 2010 December 30
}

\begin{abstract}
We present a detailed description of the Voronoi Tessellation (VT) cluster finder algorithm in $2+1$ dimensions, which improves on past implementations of this technique. The need for cluster finder algorithms able to produce reliable cluster catalogs up to redshift 1 or beyond and down to $10^{13.5}$ solar masses is paramount especially in light of upcoming surveys aiming at cosmological constraints from galaxy cluster number counts. We build the VT in photometric redshift shells and use the two-point correlation function of the galaxies in the field to both determine the density threshold for detection of cluster candidates and to establish their significance. This allows us to detect clusters in a self-consistent way without any assumptions about their astrophysical properties. We apply the VT to mock catalogs which extend to redshift 1.4 reproducing the $\Lambda \mathrm{CDM}$ cosmology and the clustering properties observed in the Sloan Digital Sky Survey data. An objective estimate of the cluster selection function in terms of the completeness and purity as a function of mass and redshift is as important as having a reliable cluster finder. We measure these quantities by matching the VT cluster catalog with the mock truth table. We show that the VT can produce a cluster catalog with completeness and purity $>80 \%$ for the redshift range up to $\sim 1$ and mass range down to $\sim 10^{13.5}$ solar masses.
\end{abstract}

Key words: cosmology: observations - galaxies: clusters: general - methods: data analysis

Online-only material: color figures

\section{INTRODUCTION}

Today we recognize that galaxies constitute a very small fraction of the total mass of a cluster, but they are nevertheless some of the clearest signposts for detection of these massive systems. Furthermore, the extensive evidence for differential evolution between galaxies in clusters and the field-and its sensitivity to the underlying cosmological model - means that it is imperative to quantify the galactic content of clusters. Perhaps even more importantly, optical detection of galaxy clusters is now inexpensive both financially and observationally. Large arrays of CCD detectors on moderate telescopes can be utilized to perform all-sky surveys with which we can detect clusters to $z \sim 1$, and even further with IR mosaics.

Forthcoming projects such as the Dark Energy Survey (DES; http://www.darkenergysurvey.org), Pan-STaRRS (http://www. pan-starrs.ifa.hawaii.edu), and the Large Synoptic Survey Telescope (LSST; http://www.lsst.org) will map thousands of square degrees to very faint limits $(\sim 29$ th magnitude per square arcsecond) in at least five filters, allowing the detection of clusters through their weak lensing signal as well as directly through the visible galaxies. Combined with ever more efficient clusterfinding algorithms, these programs will expand optical cluster detection to redshifts greater than unity. Prospects for utilization of such data to address one of the most important scientific problems of our time by measuring the cosmological parameters with improved precision are outstanding. In fact, given the statistical power of these surveys, clusters have become one of the strongest probes for dark energy (e.g., Haiman et al. 2001; Holder et al. 2001; Levine et al. 2002; Hu 2003; Rozo et al. 2007,
2010). Two unavoidable challenges imposed by these projects are to produce optimal cluster catalogs-with high completeness and purity-and to determine their selection function as a function of cluster mass and redshift.

To see how to proceed, we must understand the strengths and important limitations of the techniques in use today, especially with respect to the characterizability of the resulting catalogs. We focus on photometric techniques rather than on cluster finding in redshift space, which also has a long story, starting with Huchra \& Geller (1982), and has been successfully applied to spectroscopic redshift survey data such as 2dFGRS (Eke et al. 2004) and DEEP2 (Gerke et al. 2005). Although the Voronoi Tessellation (VT) uses redshift information, it is a photometric technique and this motivates a discussion focused on this class of cluster finders.

The earliest surveys relied on visual inspection of vast numbers of photographic plates, usually by a single astronomer. The true pioneering work in this field did not appear until the late 1950s, upon the publication of a catalog of galaxy clusters produced by Abell (1958), which remained the most cited and utilized resource for both galaxy population and cosmological studies with clusters for over 40 years. Abell et al. (1989, hereafter ACO) published an improved and expanded catalog, now including the Southern sky. These catalogs have been the foundation for many cosmological studies over the last decades, even with serious concerns about their reliability. Despite the numerical criteria laid out to define clusters in the Abell and ACO catalogs, their reliance on the human eye and use of older technology and a single filter led to various biases. These old catalogs suffered as much from being black and white as they 
did from being eye-selected. Even more disturbing, measures of completeness and contamination in the Abell catalog disagree by factors of a few. Unfortunately, some of these problems will plague any optically selected cluster sample, but the use of color information, objective selection criteria, and a strong statistical understanding of the catalog can mitigate their effects.

Only in the past 20 years has it become possible to utilize the objectivity of computational algorithms in the search for galaxy clusters. These more modern studies required that plates be digitized, so that the data are in a machine-readable form. The hybrid technology of digitized plate surveys blossomed into a cottage industry. The first objective catalog produced was the Edinburgh/Durham Cluster Catalog (Lumsden et al. 1992), which covered $0.5 \mathrm{sr}\left(\sim 1600 \mathrm{deg}^{2}\right)$ around the South Galactic Pole. Later, the APM cluster catalog (Dalton et al. 1997) was created by applying Abell-like criteria to select overdensities from the galaxy catalogs. The largest, most recent, and the last of the photo-digital cluster survey is the Northern Sky Optical Survey (Gal et al. 2000, 2003, 2009; Lopes et al. 2004). This survey relies on galaxy catalogs created from scans of the second generation Palomar Sky Survey plates, input to an adaptive kernel galaxy density mapping routine. The final catalog covers $11,733 \mathrm{deg}^{2}$, with nearly 16,000 candidate clusters, extending to $z \sim 0.3$. A supplemental catalog up to $z \sim 0.5$ was generated by Lopes et al. (2004) using VT and adaptive kernel maps.

With the advent of CCDs, fully digital imaging in astronomy became a reality. These detectors provided an order of magnitude increase in sensitivity, linear response to light, small pixel size, stability, and much easier calibration. The main drawback relative to photographic plates was (and remains) their small physical size, which permits only a small area (of order $15^{\prime}$ ) to be imaged by a larger $4096^{2}$ pixel detector. Realizing the vast scientific potential of such a survey, an international collaboration embarked on the Sloan Digital Sky Survey (SDSS, http://www.sdss.org), which included construction of a specialized $2.5 \mathrm{~m}$ telescope, a camera with a mosaic of $30 \mathrm{CCDs}$, a novel observing strategy, and automated pipelines for survey operations and data processing. Main survey operations were completed in the fall of 2005 , with over $8000 \mathrm{deg}^{2}$ of the northern sky image in five filters to a depth of $r^{\prime} \sim 22.2$ with calibration accurate to $\sim 1 \%-2 \%$, as well as spectroscopy of nearly one million objects.

With such a rich data set, many groups both internal and external to the SDSS collaboration have generated a variety of cluster catalogs, from both the photometric and the spectroscopic catalogs, using techniques including:

1. VT (Kim et al. 2002);

2. overdensities in both spatial and color space (maxBCG, Annis et al. 1999; Koester et al. 2007b; Hao 2009);

3. subdividing by color and making density maps (cut-andenhance, Goto et al. 2002);

4. the Matched Filter and its variants (Kim et al. 2002);

5. surface brightness enhancements (Zaritsky et al. 1997, 2002; Bartelmann \& White 2002);

6 . overdensities in position and color spaces, including redshifts (C4, Miller et al. 2005); and

7. friends-of-friends (FoF, Berlind et al. 2006).

Each method generates a different catalog, and early attempts to compare them have shown not only that they are quite distinct, but also that comparison of two photometrically derived cluster catalogs, even from the same galaxy catalog, is not straightforward (Bahcall et al. 2003).
In addition to the SDSS, smaller areas, but to much higher redshift, have been covered by numerous deep CCD imaging surveys. Notable examples include the Palomar Distant Cluster Survey (PDCS, Postman et al. 1996), the ESO Imaging Survey (EIS, Lobo et al. 2000), and many others. None of these surveys provide the angular coverage necessary for large-scale structure and precision cosmology studies, and have been specifically designed to find rich clusters at high redshift. The largest such survey to date is the Red Sequence Cluster Survey (Gladders $\&$ Yee 2005), based on moderately deep two-band imaging using the CFH12K mosaic camera on the Canada-France-Hawaii Telescope $3.6 \mathrm{~m}$ telescope, covers $\sim 100 \mathrm{deg}^{2}$. This area coverage is comparable to X-ray surveys designed to detect clusters at $z \sim 1$ (Vikhlinin et al. 2009).

Any cluster survey must make many different mathematical and methodological choices. Regardless of the data set and algorithm used, a few simple rules should be followed to produce a catalog that is useful for statistical studies of galaxy populations and for cosmological tests.

1. Cluster detection should be performed by an objective, automated algorithm to minimize human biases.

2. The algorithm utilized should impose minimal constraints on the physical properties of the clusters, to avoid selection biases. Any remaining biases must be properly characterized.

3. The sample selection function must be well understood, in terms of both completeness and purity, as a function of both redshift and mass. The effects of varying the cluster model on the determination of these functions must also be known.

4. The catalog should provide basic physical properties for all the detected clusters, including estimates of their distances and some mass proxy (richness, luminosity, overdensity) such that specific subsamples can be selected for future study.

One of the most popular and commonly used methods today is the VT (Ramella et al. 2001; Kim et al. 2002; Lopes et al. 2004). Our implementation of this technique is described in detail in Section 2. Briefly, it subdivides a spatial distribution into a unique set of polygonal cells, one for each object, with the cell size inversely proportional to the local density. One then defines a galaxy cluster as a high-density region, composed of small adjacent cells. VT satisfies the above criteria for generating statistical, objective, cluster samples. It requires no a priori assumption on galaxy colors, the presence of a red sequence, a specific cluster profile, or luminosity function. Mock catalogs have been used to test the efficiency of the detection algorithm. These attractive qualities have led to its employment in numerous projects beginning almost 20 years ago (van de Weygaert \& Icke 1989; Ikeuchi \& Turner 1991; van de Weygaert 1994; Zaninetti 1995; El-Ad et al. 1996; Doroshkevich et al. 1997). Ebeling \& Wiedenmann (1993) used VT to identify $\mathrm{X}$-ray sources as overdensities in X-ray photon counts. Kim et al. (2002), Ramella et al. (2001) and Lopes et al. (2004) looked for galaxy clusters using VT. van Breukelen \& Clewley (2009) included the VT as one of two methods in their 2TecX detection algorithm, an extension of their work on clusters in UKIDSS (van Breukelen et al. 2006). Barkhouse et al. (2006) used the VT to detect clusters on optical images of X-ray Chandra fields. Diehl \& Statler (2006) applied a modified version of the VT algorithm to X-ray data.

Here we improve on past implementations of this technique focusing on optical data. We build the VT in photometric 
redshift shells and use the two-point correlation function of the galaxies in the field to determine the density threshold for detection of cluster candidates and to establish their significance. This allows us to detect clusters in a self-consistent way using a minimum set of free parameters and without any assumptions about the astrophysical properties of the clusters. We provide a list of member galaxies for each cluster and use the number of members as a proxy for mass. We apply the VT on mock catalogs that accurately reproduce the $\Lambda$ CDM cosmology and the clustering properties observed in the SDSS data. By comparing the VT cluster catalog with the truth table, we measure the completeness and purity of our cluster catalog as a function of mass and redshift. We show that our implementation of the VT produces a reliable cluster catalog up to redshift $\sim 1$ and down to $\sim 10^{13.5}$ solar masses.

The paper is organized as follows: Section 2 is dedicated to a detailed presentation of the algorithm; Section 3 describes the method used to compute the selection function of the cluster catalog; in Section 4 we discuss the completeness and purity results and show our ability to recover the mass function of the mock catalog at redshift close to unity; Section 5 presents a summary of this work. The work on the relation between the two-point correlation function and the VT cell areas distribution-fundamental for the development of our method-is detailed in the Appendix.

\section{ALGORITHM}

We present the VT cluster finder in $2+1$ dimensions. The method is non-parametric and does not smooth the data, making the detection independent of the cluster shape. It uses all galaxies available, going as far down in the luminosity function as the input catalog permits. It does not rely on the existence of features such as a unique brightest cluster galaxy (BCG) or a tight ridgeline in the color-magnitude space. It works in shells of redshift, treating each shell as an independent two-dimensional field.

Central to the VT algorithm is the background over which an overdensity must rise to be identified as a cluster. In contrast to earlier implementations of the VT algorithm (Ebeling \& Wiedenmann 1993; Ramella et al. 2001; Kim et al. 2002; Lopes et al. 2004), we do not assume a Poissonian background. We use a more realistic assumption that the angular two-point correlation function of the background galaxy distribution is represented by a power law (e.g., Connolly et al. 2002). Another improvement over earlier works on VT-based cluster finders is the use of photometric redshifts instead of magnitudes (Ramella et al. 2001; Lopes et al. 2004) or colors (Kim et al. 2002). This eliminates the need for a percolation step and allows for a cluster finder which is not based on astrophysical properties of clusters (the luminosity function or color-magnitude relation), but on the characteristics of the large scale clustering process. This makes the VT a cluster finder subject to different systematics from color-based methods.

The fundamental inputs required for cluster detection using the VT are the coordinates R.A., decl., and redshift of each galaxy and the redshift error $\sigma_{z}(z)$ for the full galaxy sample. The input catalog is sliced in non-overlapping $1 \sigma_{z}$ wide redshift shells. Note that the velocity dispersion of a typical cluster is much smaller than realistic values of $\sigma_{z}$. For each shell an estimate of the parameters $(A, \gamma)$ of the two-point correlation function is required. This can be obtained directly from the data.

We then build a Voronoi diagram and compare the distribution of cell areas with the distribution expected from a background- dominated field. Since small cell size implies high density, this allows us to establish a size threshold below which the distribution is dominated by cluster members. The most significant clumps of contiguous cells smaller than this threshold are listed as clusters. This procedure is repeated on all redshift shells and the results are merged into a unique list of cluster candidates. The merge proceeds as follows. From the input galaxy catalog we extract three-dimensional boxes centered at the coordinates of each candidate. We run the VT on those boxes to confirm the detection. This recursive procedure eliminates the edge effects at the interface between successive shells, reduces the number of fake detections due to projection effects, and eliminates multiple detections.

In the resulting cluster catalog, we report position, redshift, redshift error, galaxy density contrast, significance of detection, richness, size, and shape parameters of the clusters. We also provide a list of members with the local density of their respective cells and flags indicating the central galaxy (the galaxy found in the highest density cell).

Although it is possible to build Voronoi diagrams on a sphere, we use a rectangular coordinate system, which is easier to implement. This implies that we must process small sky areas at a time to avoid distortions due to tangential projection. We have tested different area sizes and concluded that boxes of $3 \times 3$ degrees are adequate. A buffer region is implemented to avoid edge effects and the effective area is the central $1 \times 1 \mathrm{deg}^{2}$ box. Clusters found in the buffer regions are rejected prior to the merging of the shells' candidate lists. The size of the buffer zone corresponds to the angular scale of a large cluster at the lowest redshift (a $1^{\circ}$ scale corresponds to $\sim 3 \mathrm{Mpc}$ at $z=0.05$ ).

In the following, we detail each step of the cluster detection process and explain how each of the above quantities is derived, justifying the choices made in designing the algorithm.

\subsection{VT Construction}

The Voronoi diagram of a two-dimensional distribution of points is a unique, non-arbitrary, and non-parametric fragmentation of the area into polygons. A simple algorithm to perform such fragmentation is the following (see Figure 1): starting from any position $P_{1}$, we label its nearest neighbor $P_{2}$ and walk along the perpendicular bisector between those points. We stop when we reach for the first time a point $Q_{1}$ equidistant from $P_{1}, P_{2}$ and any third point $P_{3}$. We now walk along the perpendicular bisector between $P_{1}$ and $P_{3}$ until we reach the point $Q_{2}$ and identify the next point $P_{4}$ by the same criterion. Successive repetition of this process will eventually bring us back to $Q_{1}$ after a finite number of steps. The set of points $Q_{i}$ are the vertices of a polygon, the Voronoi cell, associated with $P_{1}$. If this process is repeated for each point $P_{i}$ we will have built the VT corresponding to this point field.

However, there are several more robust and efficient computational algorithms to build a Voronoi diagram from a given distribution. In our code, we use the so-called divide and conquer algorithm (D\&C) implemented in the Triangle library (Shewchuk 1996). The D\&C is based on recursive partition and local triangulation of the points and then on a merging stage. The total running time for a set of $n$ points is $O(n \log n)$.

There are no arbitrary choices in building the VT. The cell edges are segments of the perpendicular bisectors between neighbor points and each vertex is an intersection of two bisectors. This implies that the cells will be smaller in the highdensity regions and since each cell contains one and only one point, the inverse of the cell area gives the local density. The 


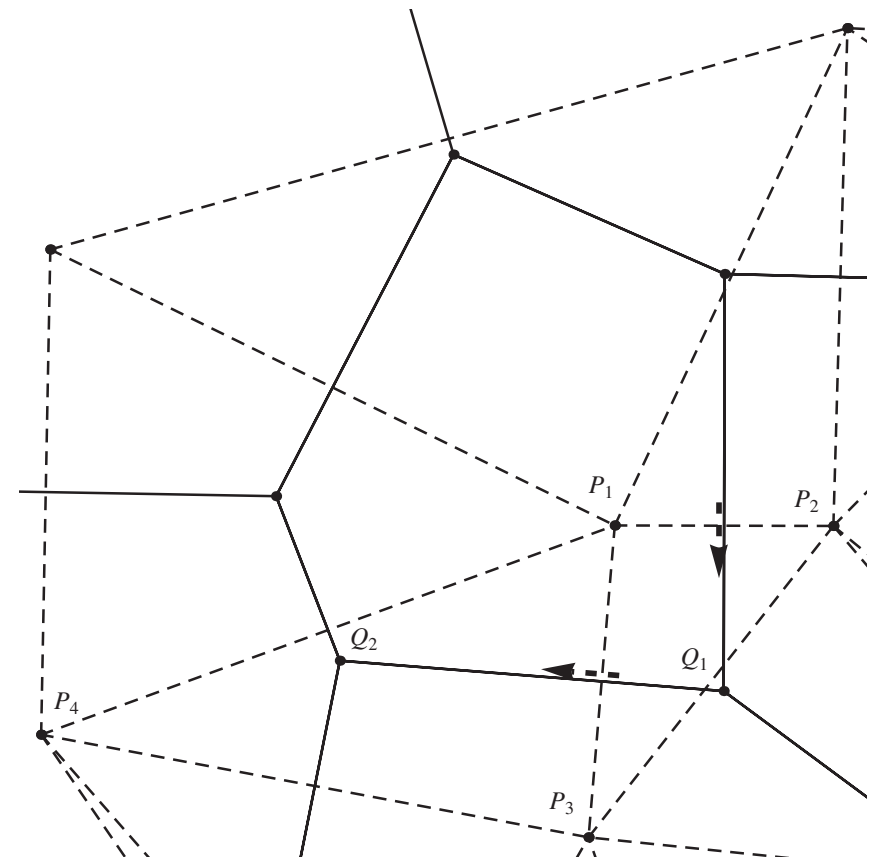

Figure 1. Portion of a typical VT is shown together with its dual Delaunay mesh (solid and dashed lines, respectively) to illustrate the Voronoi diagram building process. For each generator set $P_{i}$, there is one and only one set of Voronoi cells given by the vertices $Q_{i}$. See text for details.

VT cluster finder takes advantage of this fact in the process of detection.

\subsection{Cluster Candidate Detection}

Each realization of a given point process will result in a distinct unique tessellation, but the distribution of Voronoi cell areas will be the same. The case of the Poisson point process has been extensively investigated and it has been shown (Kiang 1966) that the resulting distribution of Voronoi cell areas is well fitted by a gamma distribution

$$
p(x)=\frac{\beta^{\alpha}}{\Gamma(\alpha)} x^{\alpha-1} \exp ^{-\beta x}
$$

with $\beta=\alpha=4$ (only for the Poisson case) and $x$ being the cell area normalized by the mean area of all cells. Here we extend Kiang's formula to a more general case.

Consider a random distribution of points in a plane with two-point correlation function given by $w(\theta)=A \theta^{1-\gamma}$, where the variable $\theta$ is the separation between point pairs and the parameters $A$ and $\gamma$ are respectively the amplitude and slope of the power law. The Poisson distribution is the particular case where $A \rightarrow 0$. A general relation between the statistics of the point field and the VT areas distribution remains as a conjecture yet to be proved, but in the case of a point field generated from the above two-point correlation function, the gamma distribution still holds with the values of $\alpha$ and $\beta$ modified. We have proven this fact and obtained the relation between $\alpha, \beta$ and the parameters $A, \gamma$ numerically. Using the simulated annealing method described in the context of materials science (Rintoul \& Torquato 1997), we generate test fields spanning a wide range of $A, \gamma$ pairs. On each test field we applied the VT algorithm and obtained the corresponding distribution of cell areas, fitting Equation (1) to obtain the corresponding pair $\alpha, \beta$. These two parameters are not independent. They are related by

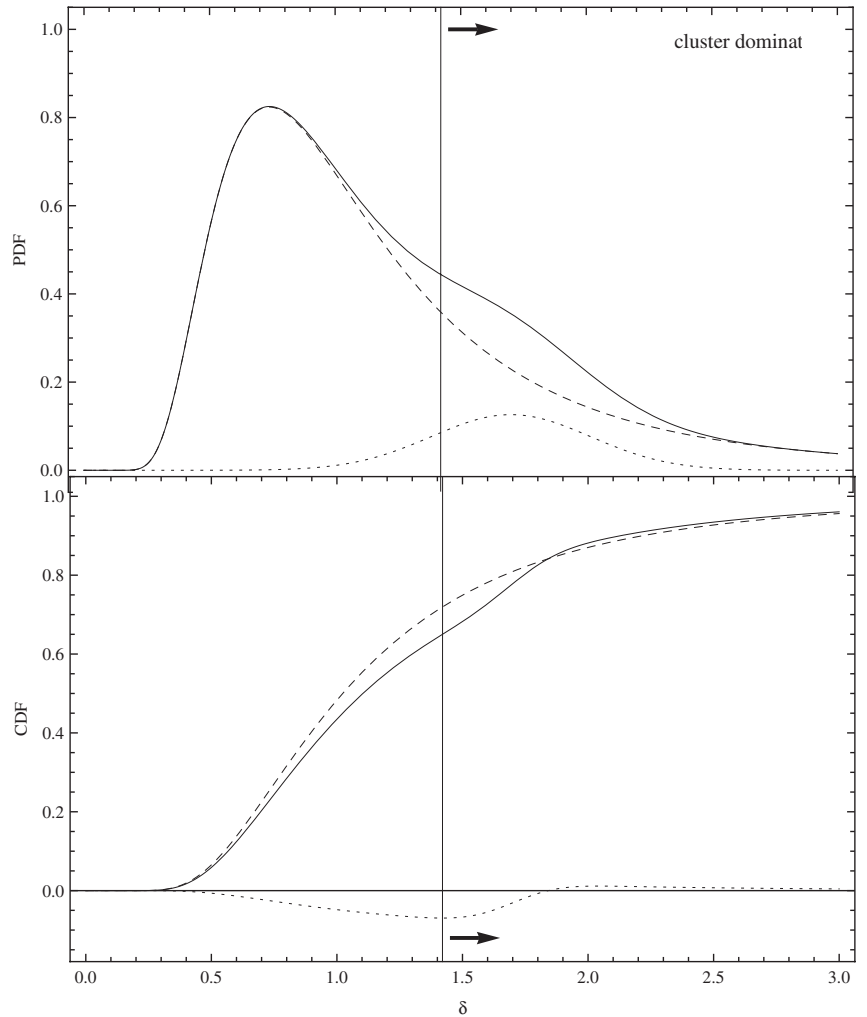

Figure 2. Differential and cumulative distributions of normalized cell densities illustrating the process of detection in the VT cluster finder. The dashed lines correspond to a background distribution with $A=0.005$ and $\gamma=1.7$. The solid lines correspond to the distributions distorted by an artificial Gaussian-shaped cluster contribution (dotted line). The vertical line is the threshold for detection $\delta^{*}$. All cells above the threshold are selected as cluster member candidates.

a simple relation: $\beta=\alpha-0.26$. See the Appendix for a detailed discussion of these results.

Information about the background is given to the VT code via the two input parameters $A, \gamma$. These will depend on the redshift shell and, ideally, they should be estimated directly from the data being considered. High accuracy in the parameters is not required, though. Note that no free parameters are introduced by $A$ and $\gamma$, since they can be completely determined from the global input galaxy catalog. Clusters and groups present in the field when the two-point correlation function is measured do not affect the cluster finder. On the contrary, our method is based on the idea that the clustering process resulting in the power law described by $A$ and $\gamma$ also results in the formation of clusters, which are found in the high-density end of the VT cell distribution.

Taking the differential probability distribution (1) as a function of the normalized cell density, $\delta=1 / x$, our goal is to identify a density threshold $\delta^{*}$ above which the contribution of the clusters starts to dominate over the background. A schematic example is shown in Figure 2. To the background distribution given by $A=0.005$ and $\gamma=1.7$ (upper panel, dashed line), we add a cluster contribution of $10 \%$ given by a simple Gaussian (upper panel, dotted line). As a result, the total distribution is distorted by the presence of the clusters. To perform the detection, we take the corresponding cumulative distributions. For the background, the cumulative distribution is given by

$$
P(\delta)=\frac{\Gamma(\alpha, \beta / \delta)}{\Gamma(\alpha)}
$$

and depends on the input parameters $A, \gamma$ through $\alpha$ and $\beta$. The 
maximum of the difference between the background (dashed) and the total (solid) distributions corresponds to the point where the total distribution increases faster than the background. This point is a natural choice for the threshold $\delta^{*}$ (vertical line).

In the example above an artificial cluster contribution with a particular shape was added to illustrate the principle of detection. In the actual process, we work only with the cumulative distributions. Once the threshold is computed we select all the cells with $\delta \geqslant \delta^{*}$. We then take the clumps of contiguous selected cells as cluster candidates.

Setting the threshold at the point of maximum difference between the two distributions leads to the detection only of the central regions of the most massive clusters $\left(M>10^{14.5} M_{\odot}\right)$. This is a consequence of the fact that the two-point correlation function of the field includes the contribution of clusters, and only the highest density peaks deviate significantly from the distribution predicted by Equation (2). To improve this result, we allow this to be an adjustable parameter, called $\mathrm{scl}$. By comparing the two-point correlation function of galaxies measured by Davis \& Peebles (1983) in the $14.5 m_{B}$ CfA redshift survey with the two-point correlation function of rich $(R \geqslant 1)$ Abell clusters measured by Bahcall \& Soneira (1983), Bahcall (1986) has estimated that $\sim 25 \%$ of all galaxies are associated with clusters and the $10 \mathrm{Mpc}$ scale structures that surround them. We therefore set our threshold at the point $\delta^{*}$ where the cumulative distribution reaches $\sim 75 \%$. As this fraction must change with redshift, magnitude limit of the galaxy catalog and lower mass limit of the cluster catalog, we determine the exact values of the cumulative distribution used to set $\delta^{*}$ in each redshift bin, $\operatorname{scl}(z)$, by applying the cluster finder on simulated galaxy catalogs and maximizing the completeness and purity of the output catalog. This process does introduce a free parameter that we must tune.

\subsection{Selection of High-significance Candidates and Membership Assignment}

For a given threshold $\delta^{*}$, we assume that each cluster candidate has a probability

$$
p\left(\delta_{\min }, N_{g}\right)=1-\operatorname{Erf}\left(\left(\frac{\delta_{\min }}{\delta^{*}}-1\right) \frac{N_{g}}{\sqrt{2}}\right)
$$

of being caused by random fluctuations of the background field. Here $\delta_{\min }$ is the minimum cell density and $N_{g}$ is the number of galaxies in the candidate. Note that the process of detection implies $\delta_{\min } \geqslant \delta^{*}$. A confidence level of $95 \%$ is required for a candidate to be accepted. If a given candidate has $p\left(\delta_{\min }, N_{g}\right)$ below this level, we iterate on its cells, dropping the one with the lowest density and recomputing $p\left(\delta_{\min }, N_{g}\right)$, until this candidate falls within the acceptable level or runs out of galaxies. As a result, some cluster candidates will be reduced in size and others will be eliminated. The final list of candidates is composed of clusters above the required confidence level. This cleaning process is necessary as the $\delta^{*}$ threshold is set to be permissive; the estimate by Bahcall (1986) that $\sim 25 \%$ of all galaxies are associated with clusters was accompanied by a hypothesis that these galaxies were distributed in $\sim 30 \mathrm{Mpc}$ scale overdense regions about clusters, while we aim to detect clusters closer to the $\sim 1 \mathrm{Mpc}$ Virial scale. This process results in a list of cluster members, given by all the galaxies within the final VT footprint of the cluster. The galaxy belonging to the cell of highest density is taken as the central galaxy.

The accuracy of the membership assignment is limited by the errors in the redshift of the galaxies and width of the redshift shell. As discussed in Section 2.5, the membership list is improved in the second run of the VT cluster finder, which is performed in boxes centered at the central galaxies flagged during this first run.

\subsection{Shape Measurement}

To obtain the cluster shape parameters, we take the galaxies within the cluster VT footprint and compute the second moments of the galaxy distribution with respect to the coordinates $\left(x_{c}, y_{c}\right)$ of the central galaxy, using the cell densities $\delta$ as weights. These second moments are

$$
\begin{gathered}
m_{x x}=\frac{\sum_{i} \delta_{i}\left(x_{i}-x_{c}\right)^{2}}{\sum_{i} \delta_{i}} \\
m_{y y}=\frac{\sum_{i} \delta_{i}\left(y_{i}-y_{c}\right)^{2}}{\sum_{i} \delta_{i}} \\
m_{x y}=\frac{\sum_{i} \delta_{i}\left(x_{i}-x_{c}\right)\left(y_{i}-y_{c}\right)}{\sum_{i} \delta_{i}}
\end{gathered}
$$

where the $x$ and $y$ directions are aligned with the R.A. and decl. axes, respectively. We use these quantities to compute the semimajor and semiminor axes, $a$ and $b$, respectively:

$$
\begin{aligned}
& a=\left[\frac{1}{2}\left(m_{x x}+m_{y y}+f\right)\right]^{1 / 2} \\
& b=\left[\frac{1}{2}\left(m_{x x}+m_{y y}-f\right)\right]^{1 / 2}
\end{aligned}
$$

where

$$
f=\left(m_{x x}-m_{y y}+4 m_{x y}\right)^{1 / 2} .
$$

The position angle is also obtained in terms of the same quantities,

$$
P A=\frac{180}{\pi} \tan ^{-1}\left(\frac{b^{2}-m_{x x}}{m_{x y}}\right),
$$

and is given in degrees.

\subsection{Catalog Construction}

A global list of cluster candidates is made by merging the results of the individual shells. For each cluster in that list we extract from the full input galaxy catalog (not the $z$ shells) a three-dimensional box centered at its central galaxy and with the same size as in the first run: $3 \times 3 \mathrm{deg}^{2}$ and $\sigma_{z}$ width. These boxes are processed with the VT algorithm, repeating the steps described in Sections 2.1-2.4, and a new global list of cluster candidates is constructed, taking only the clusters found at the center of each box.

We perform a matching between the two global lists. In this matching scheme, candidates are considered the same cluster if they have more than $50 \%$ of shared galaxies and multiple matches are not allowed. When a matching occurs, that cluster is eliminated from the list of candidates available for matching with other candidates. The clusters found in the first run but undetected in the second run are eliminated as projection effects. The primary function of this stage, however, is to deal with photo- $z$ slice edge effects. 
Because the new boxes are allowed to cross the initial shell boundaries, edge effects in the redshift dimension are eliminated. Clusters split in several components during the initial detection will result in cluster candidates with a number of shared galaxies after the second run. For a given pair of candidates found to be the same cluster (i.e., sharing more than $50 \%$ of their galaxies), only the one with the largest number of members is added to the final cluster catalog. Otherwise, they are said to be distinct clusters with shared galaxies (which are flagged in the members list) and both are included in the cluster catalog. Setting the threshold of shared galaxies to $50 \%$ is a natural choice between the two extremes where all candidates would be duplicated or only the clusters found with the same set of member galaxies would be accepted.

At this point the detection is completed. We have the final list of clusters containing R.A., decl., redshift, and a list of member galaxies including the parameters of the corresponding VT cells. This forms the VT footprint of the cluster. The cluster redshift is estimated as the median of the redshift of the cluster members. The quantity is better estimated in the second run after a cleaner membership list is obtained, so as to avoid projection effects along the line of sight.

The output parameters of the VT cluster catalog are: ID, R.A., decl. (coordinates of its central galaxy or the highest density peak), $z$ (given by the median of all members), $\sigma_{z}$ (rms value), $\delta_{c}$ (density contrast measured at the final stage of detection), $\sigma$ (significance of detection), richness (number of members), size (radius of the circle enclosing all galaxies), $a$ (semimajor axis), $b$ (semiminor axis), and P.A. (position angle).

We also report a members list containing: ID, host ID (most likely host cluster), cell density, shared flag ( 1 if the galaxy is shared with another cluster, 0 otherwise), and central flag ( 1 for central galaxy, 0 for regular members). Note that we do not list every possible galaxy-cluster association in the output. Galaxies not associated to any cluster are listed with host ID, shared flag and central flag set to -1 . These non-member galaxies can be used, for instance, to compute the local density of non-member galaxies around a cluster or to run afterburners to measure cluster properties such as richness and $R_{200}$.

Having a list of members generated by the cluster finder is highly desirable, because properties such as the optical richness and $R_{200}$ can be estimated. The lack of membership assignment in VT implementations using magnitudes was a drawback and we improve on that matter. Also, this allows us to compute the algorithm efficiency as follows.

\section{ALGORITHM EFFICIENCY}

The effectiveness of the algorithm is evaluated by measuring the VT catalog completeness and purity as a function of mass and redshift. These quantities are the selection function needed to understand the catalog. The completeness and purity are best measured with mock galaxy catalogs with known relations to dark matter halos. The field can no longer be advanced by placing single clusters in the center of an image with random backgrounds.

We apply the algorithm to a mock galaxy catalog and match the resulting cluster catalog with the corresponding mock truth table of halos-the truth table. This allows us to define completeness as the fraction of halos with a VT cluster counterpart and purity as the fraction of VT clusters with a matching halo. We perform this in bins of redshift and we also estimate the impact of redshift errors.

\subsection{Mock Catalogs}

Mock galaxy catalogs are created using the ADDGALS code (Busha \& Wechsler 2008; Wechsler 2004; see also Gerdes et al. 2010, Appendix A). ADDGALS takes an $N$-body simulation light cone and attaches galaxies to its dark matter particles to create a deep mock photometric catalog using an $N$-body simulation with only modest mass resolution. The resulting galaxy catalog reproduces the luminosity function, the magnitude-dependent two-point correlation function, and the color-density-luminosity distribution measured from the SDSS data. The mock catalogs used here were based on the Hubble volume simulation that modeled a $3 \mathrm{Gpc} h^{-1}$ box with $1024^{3}$ particles in a flat $\Lambda \mathrm{CDM}$ cosmology with $\Omega_{M}=0.3$ and $\sigma_{8}=0.9$ (Evrard et al. 2002).

ADDGALS first builds a list of galaxies $r$-band luminosities drawing from a luminosity function $\phi\left(M_{r}\right)$, and assigns these galaxies to individual dark matter particles in the simulation. Here, $\phi\left(M_{r}\right)$ is the observed SDSS $r$-band luminosity function at redshift $\sim 0.1$ from Blanton et al. (2003) assuming passive evolution of $1.3 \mathrm{mag}$ per unit redshift. These galaxies are then mapped to individual dark matter particles using a probability relation $P\left(R_{\delta} \mid L_{r} / L_{*}\right)$ that relates to local dark matter overdensity to the luminosity of a galaxy. Overdensities of dark matter are computed using the characteristic radius $R_{\delta}$, defined as the radius enclosing $1.8 \times 10^{13} h^{-1}$ solar masses of dark matter. The form of $P\left(R_{\delta} \mid L_{r} / L_{*}\right)$ is taken to be a Gaussian plus a log-normal representing galaxies in the "field," i.e., unresolved low-mass halos, and those in higher mass, well-resolved "halos." The exact form of this relation is

$$
\begin{aligned}
& P\left(R_{\delta} l \mid L_{r} / L_{*}\right)=\frac{(1-p(L))}{R \sqrt{2 \pi} \sigma_{c}\left(L_{r} / L_{*}\right)} \\
& \times e^{-\left(\ln \left(R_{\delta} l\right)-\mu_{c}\left(L_{r} / L_{*}\right)\right)^{2} / 2 \sigma_{c}\left(L_{r} / L_{*}\right)^{2}} \\
& +\frac{p\left(L_{r} / L_{*}\right)}{\sqrt{2 \pi} \sigma_{f}\left(L_{r} / L_{*}\right)} e^{\left(R_{\delta}-\mu_{f}\left(L_{r} / L_{*}\right)\right)^{2} / 2 \sigma_{f}\left(L_{r} / L_{*}\right)^{2}} .
\end{aligned}
$$

The exact values of the parameters for this function are determined using a Monte Carlo Markov chain analysis, imposing that the observed magnitude-dependent two-point correlation function is matched.

The next step is to assign galaxy colors. The local galaxy density is computed for each galaxy in the simulation and in a training set of galaxies from the magnitude-limited SDSS DR6 catalog using the projected distance to the fifth nearest neighbor in a bin of redshift as in Cooper et al. (2007). Each mock galaxy is assigned the spectral energy distribution (SED) of a randomly selected SDSS galaxy with similar local galaxy density and absolute magnitude $M_{r}$. When doing this matching, we do not match absolute measurements of the densities, but instead opt for a relative matching where the SEDs from the densest galaxies in our training set are matched to the densest galaxies in the mock. This lets up more robustly assign SEDs to higher redshift objects where our training set is incomplete. The SED is then $k$-corrected and the appropriate filters are applied to obtain SDSS colors. At high redshift, color information is extrapolated from low redshifts: $r$-band magnitudes are passively evolved before selecting the SED from our training-set galaxy which is then $k$-corrected assuming that the rest-frame colors and the color-density-luminosity distribution remain unchanged.

The resulting catalog reproduces the overall photometric and clustering properties of the SDSS galaxies at low redshifts 
$(z \sim 0.3)$ and extends, using simplified assumptions, to higher redshifts $(z \sim 1.3)$ and deeper magnitudes $(r \sim 24)$. The BCGs, however, are an exception. BCGs luminosities are tightly correlated with their host halo mass and are not reproduced by this method. Therefore, a BCG luminosity is calculated for each resolved halo (of mass $\sim 5 \times 10^{13} h^{-1} M_{\odot}$ and above) using the measurements from Hansen et al. (2005) before the usual galaxy-to-dark-matter particle assignment begins. The corresponding galaxies are then removed from the initial list of galaxies and placed at the center of its host halo.

We run our cluster finder on the mock catalog and compare our results with the truth table. The quantities featured in the truth table are R.A., decl., redshift and $M_{200}$, plus list of member galaxies of each halo. In this paper, we refer to the truth table as the halo catalog, and to the VT output as the cluster catalog. The quantities we use as inputs are: R.A., decl., and photometric redshift. We generate photometric redshifts from the true redshifts, using a Gaussian distribution of width $\sigma_{z}(1+z)$. We test four different values of $\sigma_{z}$, namely 0.015 , $0.03,0.045$, and 0.06 , to access the impact of the photometric redshift errors in our cluster finder.

The discussion so far was restricted to a perfect volume limited galaxy catalog. A real galaxy catalog, however, will have an irreducible level of contamination and incompleteness. Here we mimic the effects of these two quantities in the mocks by assuming that the input galaxy catalog has a completeness function given by a Fermi-Dirac distribution

$$
C_{g}(r)=\frac{f_{0}}{1+\exp ((r-\mu) / \sigma)}
$$

where $\mu$ is the magnitude limit of the catalog, $f_{0}$ is a normalization constant, and the parameter $\sigma$ controls how fast the completeness falls when the magnitude limit is reached. The parameters $f_{0}$ and $\sigma$ are taken from processing of the SDSS data with the 2DPHOT package (La Barbera et al. 2008). We found that $f_{0}=0.99$ and $\sigma=0.2$ are typical values. We degrade the mock catalogs using $\mu=23.5$, interpreting $C_{g}(r)$ as the probability that a galaxy of magnitude $r$ is detected. Similarly, from the SDSS data we infer that a small fraction of contaminants, due to misclassified stars, can be present in the input catalog. The fraction of misclassified objects increases exponentially for magnitudes above $\mu-1.5$. We take this fact into account by generating false galaxies randomly above this limit and drawing from (8) the probability that this object is actually added to the catalog.

\subsection{Membership Matching}

The evaluation of completeness and purity requires a welldefined matching scheme between the cluster catalog and the truth table. We use a membership-based matching method. Membership matching has been used in evaluating completeness and purity of both photometric and spectroscopic catalogs (White \& Kochanek 2002; Eke et al. 2004; Gerke et al. 2005; Koester et al. 2007a). Unlike cylindrical matching, which has been largely employed in this kind of study, this method is parameter-free, unambiguous and provides the means to evaluate the efficiency of the cluster finder as a function of halo mass regardless of the observable proxy for mass. This allows us to distinguish the aspects relevant to the cluster finding problem from aspects connected to the mass-observable proxy calibration, which is a problem per se and is better addressed by a separate set of post-finding algorithms.
The inputs for the matching code are the halo catalog and the cluster catalog. The first is ranked by mass, while the latter is ranked by the number of galaxies, both in descending order and in bins of redshift. It is critical to do the ranking in bins of redshift for both the halos and the clusters. In the case of halos, the mass function is evolving, so the masses will be changing at fixed rank. In the case of the clusters, the flux limit forces a changing luminosity limit with redshift, so the ranks will be changing at fixed mass. If this is not taken into account, a massive cluster at high $z(z \sim 1)$ will get a much lower rank than a massive cluster at low $z(z \sim 0.1)$.

After ranking, the first step is to fit a rank-mass relation $R(M)$ to the cluster catalog, provided rank, and the matched halo catalog provided mass. We use the fitting formula

$$
R(M)=\left(\frac{M}{M_{p}}\right)^{\alpha} \exp \left(\exp \left(\frac{M_{0}-M}{M_{e}}\right)-\frac{M}{M_{1}}\right) .
$$

This relation has no motivation other than a global fitting function, valid at all redshifts provided that the ranking is performed as described above. For our mock catalogs, the bestfit parameters for this fitting function are $M_{p}=2.26 \times 10^{17}$, $M_{e}=1.40 \times 10^{14}, M_{0}=1.85 \times 10^{13}, M_{1}=1.85 \times 10^{14}$, and $\alpha=-1.15$. We then invert the relation above to compute an "observed mass" for each cluster and proceed to the matching. If the proxy used to rank the clusters has a tight correlation with mass, the ranking will be accurate and the observed mass will show a tight correlation with the true mass for the matched pairs. It is important to notice that the use of ranking instead of observed mass does not require the mass-observable relation to be calibrated. Moreover, neither mass information nor the ranking is used in the matching process, which is membership based.

A match takes place if a fraction of member galaxies is shared by a halo-cluster pair. The best match is the object sharing the largest fraction of galaxies. We require unique matching, in which a given halo/cluster is not allowed to be associated with more than one cluster/halo. As both lists are ranked by number of galaxies, uniqueness is imposed by eliminating a matched object from the list of available objects for future matches down the list. We also require two-way matching, where the best matching pair is found when the matching is performed in both directions, halos-to-clusters and clusters-to-halos.

We note that this approach to cluster-halo matching is quite general and can be applied to any cluster-finding algorithm that produces a list of cluster members. It will be developed in more detail as a framework for comparing different algorithms establishing their usefulness for cosmological tests (B. Gerke et al. 2011, in preparation).

\subsection{Completeness and Purity}

Completeness is defined as the fraction of halos having a counterpart in the cluster catalog. Purity in turn is defined as the fraction of objects in the cluster catalog that correspond to a true halo. In both cases, only unique two-way matches are considered. Allowing for non-unique matching, where each cluster may have more than one matching halo and vice versa, would be a more permissive approach. For instance, purity would not be affected by a halo being split in two components and completeness would not be affected by two halos appearing as a single cluster. 
We count the number of matched objects in bins of mass and redshift. Therefore,

$$
\begin{aligned}
& C(M, z)=\frac{N_{\text {matched }}(M, z)}{N_{\text {halos }}(M, z)} \\
& P(M, z)=\frac{N_{\text {matched }}(M, z)}{N_{\text {clusters }}(M, z)} .
\end{aligned}
$$

Note that $C(M, z)$ can be computed using the true mass of the halos, being totally independent of the mass proxy used to rank the clusters. The true mass of the clusters, however, is available only for the matched objects. Therefore $P(M, z)$ has to be computed using the observed mass and does depend on the ranking. We fit a power law to the $M_{\mathrm{obs}}-M_{\text {true }}$ relation from the matched objects and use it to transform the scale in the $P(M, z)$ plots and show both completeness and purity as a function of $M_{\text {true }}$. This cannot be performed before the rank-mass relation fitting step, which is part of the matching process. This method allow us to evaluate the efficiency of any cluster finder imposing minimum requirements, namely a list of members for each cluster. The selection function can be defined in terms of completeness and purity as

$$
f(M, z)=\frac{C(M, z)}{P(M, z)} .
$$

This is a simplified definition. For cosmological studies with real data, $f(M, z)$ should be defined and evaluated in a likelihood analysis that includes the scatter in the mass-observable relation after calibration. Here, however, we simply want to compare the observed cluster number counts $N_{\text {obs }}(M, z)$ to the predictions from the $\Lambda \mathrm{CDM}$ cosmological model $N_{\Lambda \mathrm{CDM}}(M, z)$. In this case, the selection function is easily taken into account:

$$
N_{\text {obs }}(M, z)=f(M, z) N_{\Lambda \mathrm{CDM}}(M, z) .
$$

This comparison allows us to develop a feel for how well we can recover the true cluster number counts using the VT catalog and our ability to perform a cosmological test using VT clusters as a probe.

The method described above is very simplified with respect to the procedures involved in an actual measurement of the mass function. This would require a measurement of the mass-observable relation and its scatter. We do not perform this because the VT cluster catalog provides only Ngals, the number of galaxies on the membership list, as a mass proxy. This Ngals was not optimized to have a tight relation with mass, such as, for example, the $\lambda$ estimator of Rozo et al. (2009). Measuring and optimizing a mass proxy is a necessary step if the VT is to be used in performing cosmological tests. But this problem is better addressed by a separate algorithm, specifically designed to provide a calibrated mass proxy including the mean relation and the scatter.

\section{RESULTS AND DISCUSSION}

In Figure 3, we show the completeness and purity as a function of mass and redshift for different Gaussian $\sigma_{z}$ values. The photometric redshift errors have a strong impact on both completeness and purity. For $\sigma_{z}=0.015$, completeness lies above $80 \%$ for all redshift bins and masses above $\sim 10^{13.5} M_{\odot}$. Purity, however, drops significantly at the low-mass end. We attribute this to the fact that the range $10^{13.5}-10^{14} M_{\odot}$ is at the lower boundary of the halo catalogs associated with the mock catalog. ADDGALS will populate some fraction of real dark matter clumps in the simulation even if they are below the threshold for detection in the halo catalog. A fraction of these halos were populated with galaxies by ADDGALS, but were not listed in the truth table. We have no means to determine the exact fraction at this point and therefore we interpret the purity curve as a lower limit.

In the high-redshift regime, completeness and purity do not change much with $\sigma_{z}$. The lowest redshift bin, however, shows the lowest purity and completeness in almost all cases. This might be due to the large angular size of clusters at low $z$, as at $z \sim 0.1$ the target area of $1 \mathrm{deg}^{2}$ corresponds to only a few times the typical $R_{200}$. However, even in this case the VT catalog achieves completeness and purity above $\sim 80 \%$ at all masses. Since we are most interested in a reliable catalog at high redshifts, we consider the cluster finder efficiency, as shown in Figure 3, very good.

Note that the behavior of purity is qualitatively different in the last panel, $\sigma_{z}=0.060$. This may be connected to low-redshift clusters leaking to high redshift shells at higher rates than the high redshift ones fall toward low redshift.

Testing the effect of changes in the cluster finder free parameters on the completeness and purity functions, we find the following.

1. Changing the fraction of shared galaxies required to consider two candidates as the same cluster in the range $40 \%-60 \%$ has less than $1 \%$ impact on the results. We fix this value at $50 \%$.

2. The selection function is very sensitive to $\operatorname{scl}(z)$. Setting $\operatorname{scl}(z)$ too high $(>0.97)$ leads to fragmentation of clusters, which affects purity at all masses, and failure to detect low contrast clusters, which affects completeness at the low-mass end. Setting $\operatorname{scl}(z)$ below 0.75 causes merging of clusters and affects completeness. An optimal value for $\operatorname{scl}(z)$ in the range $0.75-0.97$ has to be found at each redshift bin.

3. The confidence level threshold has little effect on the detection. The final list of clusters shows less than $10 \%$ difference when this parameter varies in the range $90 \%-99.5 \%$. But it affects the selection function by modifying the membership list.

Figure 4 illustrates our ability to recover the true cluster number counts of the input catalog. We take the case $\sigma_{z}=$ $0.015(1+z)$ and the redshift bin $0.9<z<1.1$. For a given mass bin $M_{i}$ we divide the number of VT clusters detected by the selection function term $f\left(M_{i}, z\right)$. We then sum the corrected counts through all bins of mass $>M$ (red solid line). The curve for the truth table is done by counting all the halos above $M$ (black dotted line). We finally plot (blue dashed line) the values expected in a $\Lambda \mathrm{CDM}$ cosmology (e.g., Evrard et al. 2002) for comparison.

There is a remarkable agreement between the three curves. The tilt of the measured curve with respect to the truth table may be interpreted as low-mass clusters being misplaced toward more massive bins, due to our neglect of the scatter in the mass-observable relation. As pointed out in Section 3.3, the method used here does not take into account crucial steps involved in an actual measurement of the mass function. This issue must be addressed with a full program of mass calibration and is beyond the scope of this paper. The result shown in Figure 4 encourages the pursuit of such a program, though. Our results show that the VT is a reliable cluster finder in the 


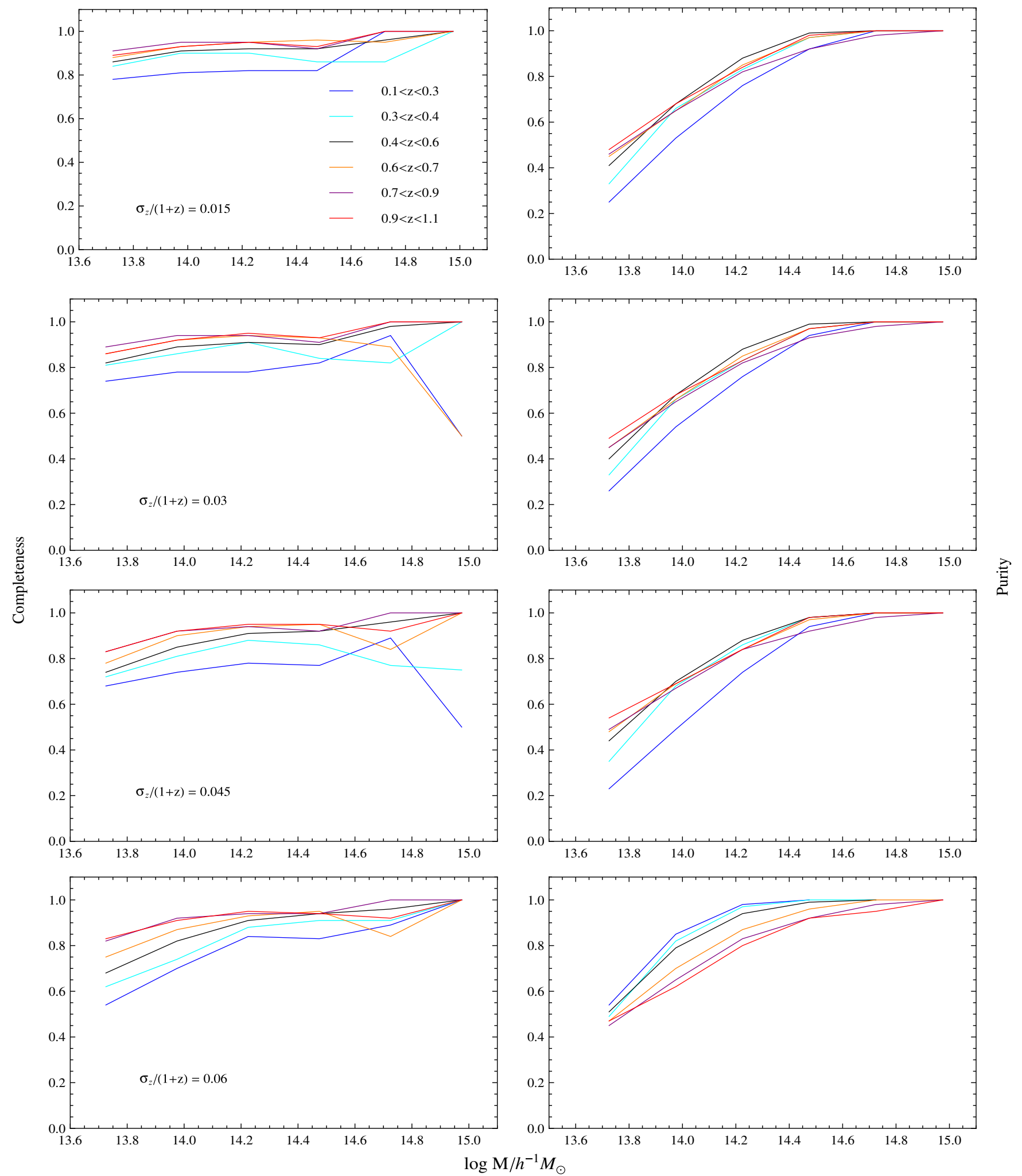

Figure 3. Completeness (left) and purity (right) curves as a function of mass for six redshift bins: $0.1<z<0.3$ (blue), $0.3<z<0.4$ (cyan), $0.4<z<0.6$ (black), $0.6<z<0.7$ (orange), $0.7<z<0.9$ (purple), $0.9<z<1.1$ (red). From top to bottom, the plot pairs feature different $\sigma_{z}$ values: $0.015,0.03,0.045,0.06$. The photometric redshift errors have a strong impact on both completeness and purity. In the best case, completeness and purity rest above $80 \%$ for all redshift bins and masses above $\sim 10^{14.2}$. In the case of purity, this curve should be interpreted as a lower limit (see text for discussion).

(A color version of this figure is available in the online journal.)

redshift and mass range of interest, as seen in the completeness and purity curves. Application of this algorithm on SDSS data is underway and will be presented in a forthcoming paper (M. Soares-Santos et al. (2011, in preparation). 


\section{SUMMARY}

In this paper, we present an improved implementation of the VT cluster finder. Improvements with respect to earlier works include the following:

1. the use of photometric redshifts instead of magnitudes;

2. a more realistic assumption that galaxy fields have twopoint correlation function described by a power law, and not by a Poisson distribution; and

3. implementation of a membership assignment scheme.

The VT cluster finder in $2+1$ dimensions was tailored to fulfill the requirements of upcoming cosmological experiments aiming at using clusters as probes for dark energy. The main challenges toward this goal include the construction of reliable cluster catalogs up to high redshifts $(z \sim 1)$ and down to lowmass limits $\left(\sim 10^{13.5} M_{\odot}\right)$ and the measurement of the selection function as a function of $M$ and $z$. To achieve these goals using the VT we:

1. adapted the VT algorithm to use photometric redshift shells and take advantage of the relation that we have discovered between the two-point correlation function of the galaxy field and its distribution of VT cell areas;

2. defined the selection function in term of completeness and purity, establishing an objective way to measure these quantities using simulated catalogs;

3. applied the VT to mock galaxy catalogs and computed the completeness and purity of the output cluster catalog with the truth table, showing that the VT can produce cluster catalogs with completeness and purity above $80 \%$ in the ranges of interest within the $M-z$ parameter space; and

4. computed the cluster abundance from the VT catalog and compared it to the halo abundance in the mocks, finding a remarkable agreement at all mass bins.

These results allow us to be confident in our ability to perform a cosmological test for dark energy using the VT algorithm on a data set of sufficient scope. Analysis of the application of the VT to the SDSS data is underway and will be presented elsewhere.

M.S.-S. has received support from the Brazilian agency $\mathrm{CNPq}$ and from the Fermilab Center for Particle Astrophysics for this work. R.H.W. and B.F.G. received support from the US Department of Energy under contract number DE-AC0276SF00515. Thanks to Massimo Ramella for making his code available at http://www.ts.astro.it/astro/VoroHome/ and to Yang Jiao for pointing out the simulated annealing method applied in this paper. Thanks to Jorge Horvath for careful reading of the manuscript.

\section{APPENDIX \\ THE VORONOI TESSELLATION CELL AREAS DISTRIBUTION FOR POWER-LAW CORRELATED POINT PROCESSES}

Motivated by what is known about the two-point correlation function of galaxies in the universe, we consider a twodimensional point field characterized by a two-point correlation function of the form

$$
w(\theta)=A \theta^{1-\gamma},
$$

where $\theta$ is a distance, $A$ is the amplitude of the correlation, and $\gamma$ is the slope of the power law. $A=0$ represents the

Table 1

VT Cell Area Distribution Model Parameters

\begin{tabular}{|c|c|c|c|c|c|}
\hline$A$ & $\gamma$ & & $\alpha$ & $\beta$ & $\chi^{2} / v$ \\
\hline 0.001 & 1.0 & $\ldots$ & $3.91 \pm 0.05$ & $3.66 \pm 0.05$ & 1.24 \\
\hline 0.001 & 1.1 & $\ldots$ & $3.86 \pm 0.05$ & $3.61 \pm 0.05$ & 1.49 \\
\hline 0.001 & 1.2 & $\ldots$ & $3.93 \pm 0.04$ & $3.68 \pm 0.03$ & 1.12 \\
\hline 0.001 & 1.3 & $\ldots$ & $3.92 \pm 0.04$ & $3.67 \pm 0.03$ & 1.74 \\
\hline 0.001 & 1.4 & $\ldots$ & $3.81 \pm 0.04$ & $3.57 \pm 0.04$ & 1.7 \\
\hline 0.001 & 1.5 & $\ldots$ & $3.96 \pm 0.04$ & $3.71 \pm 0.03$ & 1.33 \\
\hline 0.001 & 1.6 & $\ldots$ & $3.91 \pm 0.04$ & $3.65 \pm 0.03$ & 1.25 \\
\hline 0.001 & 1.7 & $\ldots$ & $3.86 \pm 0.05$ & $3.62 \pm 0.05$ & 2.07 \\
\hline 0.001 & 1.8 & $\ldots$ & $3.81 \pm 0.04$ & $3.57 \pm 0.03$ & 1.49 \\
\hline 0.001 & 1.9 & $\ldots$ & $3.94 \pm 0.02$ & $3.69 \pm 0.01$ & 1.19 \\
\hline 0.002 & 1.0 & $\ldots$ & $3.94 \pm 0.04$ & $3.71 \pm 0.03$ & 1.78 \\
\hline 0.002 & 1.1 & $\ldots$ & $3.87 \pm 0.05$ & $3.63 \pm 0.04$ & 1.18 \\
\hline 0.002 & 1.2 & $\ldots$ & $3.93 \pm 0.04$ & $3.69 \pm 0.05$ & 1.61 \\
\hline 0.002 & 1.3 & $\ldots$ & $3.87 \pm 0.02$ & $3.63 \pm 0.03$ & 2.17 \\
\hline 0.002 & 1.4 & $\ldots$ & $3.83 \pm 0.04$ & $3.58 \pm 0.03$ & 1.43 \\
\hline 0.002 & 1.5 & $\ldots$ & $3.9 \pm 0.04$ & $3.66 \pm 0.03$ & 1.4 \\
\hline 0.002 & 1.6 & $\ldots$ & $3.95 \pm 0.04$ & $3.7 \pm 0.03$ & 1.36 \\
\hline 0.002 & 1.7 & $\ldots$ & $3.79 \pm 0.04$ & $3.55 \pm 0.04$ & 1.41 \\
\hline 0.002 & 1.8 & $\ldots$ & $3.84 \pm 0.04$ & $3.59 \pm 0.03$ & 1.57 \\
\hline 0.002 & 1.9 & $\ldots$ & $3.89 \pm 0.04$ & $3.65 \pm 0.05$ & 1.57 \\
\hline 0.003 & 1.0 & $\ldots$ & $3.81 \pm 0.04$ & $3.56 \pm 0.04$ & 1.49 \\
\hline 0.003 & 1.1 & $\ldots$ & $3.9 \pm 0.04$ & $3.65 \pm 0.05$ & 1.22 \\
\hline 0.003 & 1.2 & $\ldots$ & $3.94 \pm 0.04$ & $3.69 \pm 0.03$ & 1.51 \\
\hline 0.003 & 1.3 & $\ldots$ & $3.84 \pm 0.04$ & $3.6 \pm 0.03$ & 1.62 \\
\hline 0.003 & 1.4 & $\ldots$ & $3.86 \pm 0.01$ & $3.61 \pm 0.01$ & 1.53 \\
\hline 0.003 & 1.5 & $\ldots$ & $3.97 \pm 0.04$ & $3.72 \pm 0.03$ & 1.29 \\
\hline 0.003 & 1.6 & $\ldots$ & $3.81 \pm 0.04$ & $3.57 \pm 0.04$ & 1.89 \\
\hline 0.003 & 1.7 & $\ldots$ & $3.8 \pm 0.04$ & $3.55 \pm 0.05$ & 2.03 \\
\hline 0.003 & 1.8 & $\ldots$ & $3.81 \pm 0.05$ & $3.57 \pm 0.04$ & 1.49 \\
\hline 0.003 & 1.9 & $\ldots$ & $3.86 \pm 0.04$ & $3.61 \pm 0.05$ & 1.56 \\
\hline 0.004 & 1.0 & $\ldots$ & $3.86 \pm 0.04$ & $3.62 \pm 0.05$ & 1.59 \\
\hline 0.004 & 1.1 & $\ldots$ & $3.81 \pm 0.04$ & $3.56 \pm 0.05$ & 1.47 \\
\hline 0.004 & 1.2 & $\ldots$ & $3.79 \pm 0.04$ & $3.55 \pm 0.04$ & 1.35 \\
\hline 0.004 & 1.3 & $\ldots$ & $3.87 \pm 0.04$ & $3.62 \pm 0.03$ & 1.65 \\
\hline 0.004 & 1.4 & $\ldots$ & $3.85 \pm 0.04$ & $3.6 \pm 0.05$ & 1.42 \\
\hline 0.004 & 1.5 & $\ldots$ & $3.97 \pm 0.04$ & $3.73 \pm 0.03$ & 1.24 \\
\hline 0.004 & 1.6 & $\ldots$ & $3.87 \pm 0.05$ & $3.63 \pm 0.05$ & 1.35 \\
\hline 0.004 & 1.7 & $\ldots$ & $3.82 \pm 0.04$ & $3.57 \pm 0.04$ & 1.38 \\
\hline 0.004 & 1.8 & $\ldots$ & $3.91 \pm 0.04$ & $3.66 \pm 0.03$ & 1.04 \\
\hline 0.004 & 1.9 & $\ldots$ & $3.9 \pm 0.02$ & $3.65 \pm 0.01$ & 1.33 \\
\hline 0.005 & 1.0 & $\ldots$ & $3.81 \pm 0.01$ & $3.56 \pm 0.03$ & 1.51 \\
\hline 0.005 & 1.1 & $\ldots$ & $3.86 \pm 0.04$ & $3.61 \pm 0.03$ & 1.53 \\
\hline 0.005 & 1.2 & $\ldots$ & $3.85 \pm 0.04$ & $3.6 \pm 0.05$ & 1.61 \\
\hline 0.005 & 1.3 & $\ldots$ & $3.8 \pm 0.04$ & $3.55 \pm 0.04$ & 1.41 \\
\hline 0.005 & 1.4 & $\ldots$ & $3.83 \pm 0.04$ & $3.58 \pm 0.03$ & 1.71 \\
\hline 0.005 & 1.5 & $\ldots$ & $3.87 \pm 0.04$ & $3.63 \pm 0.05$ & 1.25 \\
\hline 0.005 & 1.6 & $\ldots$ & $3.81 \pm 0.04$ & $3.57 \pm 0.03$ & 1.14 \\
\hline 0.005 & 1.7 & $\ldots$ & $3.89 \pm 0.04$ & $3.65 \pm 0.05$ & 1.16 \\
\hline 0.005 & 1.8 & $\ldots$ & $3.96 \pm 0.04$ & $3.69 \pm 0.05$ & 1.84 \\
\hline 0.005 & 1.9 & $\ldots$ & $3.88 \pm 0.04$ & $3.64 \pm 0.05$ & 1.56 \\
\hline 0.006 & 1.0 & $\ldots$ & $3.9 \pm 0.05$ & $3.66 \pm 0.04$ & 1.56 \\
\hline 0.006 & 1.1 & $\ldots$ & $3.78 \pm 0.01$ & $3.54 \pm 0.03$ & 1.47 \\
\hline 0.006 & 1.2 & $\ldots$ & $3.84 \pm 0.04$ & $3.61 \pm 0.03$ & 1.13 \\
\hline 0.006 & 1.3 & $\ldots$ & $3.88 \pm 0.05$ & $3.63 \pm 0.05$ & 1.48 \\
\hline 0.006 & 1.4 & $\ldots$ & $3.83 \pm 0.01$ & $3.59 \pm 0.01$ & 2. \\
\hline 0.006 & 1.5 & $\ldots$ & $3.86 \pm 0.04$ & $3.61 \pm 0.05$ & 1.62 \\
\hline 0.006 & 1.6 & $\ldots$ & $3.71 \pm 0.04$ & $3.47 \pm 0.04$ & 2.34 \\
\hline 0.006 & 1.7 & $\ldots$ & $3.86 \pm 0.02$ & $3.61 \pm 0.03$ & 1.62 \\
\hline 0.006 & 1.8 & $\ldots$ & $3.92 \pm 0.05$ & $3.67 \pm 0.05$ & 1.34 \\
\hline 0.006 & 1.9 & $\ldots$ & $3.91 \pm 0.02$ & $3.66 \pm 0.01$ & 1.25 \\
\hline 0.007 & 1.0 & $\ldots$ & $3.85 \pm 0.05$ & $3.6 \pm 0.04$ & 1.53 \\
\hline 0.007 & 1.1 & $\ldots$ & $3.9 \pm 0.04$ & $3.64 \pm 0.05$ & 2.08 \\
\hline 0.007 & 1.2 & $\ldots$ & $3.84 \pm 0.01$ & $3.6 \pm 0.03$ & 1.13 \\
\hline 0.007 & 1.3 & $\ldots$ & $3.82 \pm 0.04$ & $3.57 \pm 0.03$ & 1.51 \\
\hline 0.007 & 1.4 & $\ldots$ & $3.89 \pm 0.02$ & $3.64 \pm 0.001$ & 1.43 \\
\hline 0.007 & 1.5 & $\ldots$ & $3.81 \pm 0.01$ & $3.56 \pm 0.01$ & 2.11 \\
\hline
\end{tabular}


Table 1

(Continued)

\begin{tabular}{|c|c|c|c|c|c|}
\hline$A$ & $\gamma$ & & $\alpha$ & $\beta$ & $\chi^{2} / \nu$ \\
\hline 0.007 & 1.6 & $\ldots$ & $3.84 \pm 0.05$ & $3.59 \pm 0.05$ & 1.53 \\
\hline 0.007 & 1.7 & $\ldots$ & $3.77 \pm 0.01$ & $3.52 \pm 0.001$ & 1.29 \\
\hline 0.007 & 1.8 & $\ldots$ & $3.75 \pm 0.04$ & $3.5 \pm 0.04$ & 1.91 \\
\hline 0.007 & 1.9 & $\ldots$ & $3.86 \pm 0.05$ & $3.61 \pm 0.04$ & 1.52 \\
\hline 0.008 & 1.0 & $\ldots$ & $3.86 \pm 0.04$ & $3.61 \pm 0.05$ & 1.89 \\
\hline 0.008 & 1.1 & $\ldots$ & $3.87 \pm 0.04$ & $3.62 \pm 0.05$ & 1.53 \\
\hline 0.008 & 1.2 & $\ldots$ & $3.92 \pm 0.05$ & $3.68 \pm 0.05$ & 1.67 \\
\hline 0.008 & 1.3 & $\ldots$ & $3.82 \pm 0.04$ & $3.57 \pm 0.03$ & 1.32 \\
\hline 0.008 & 1.4 & $\ldots$ & $3.83 \pm 0.04$ & $3.59 \pm 0.03$ & 1.66 \\
\hline 0.008 & 1.5 & $\ldots$ & $3.99 \pm 0.04$ & $3.73 \pm 0.03$ & 1.67 \\
\hline 0.008 & 1.6 & $\ldots$ & $3.86 \pm 0.02$ & $3.61 \pm 0.03$ & 1.46 \\
\hline 0.008 & 1.7 & $\ldots$ & $3.88 \pm 0.05$ & $3.63 \pm 0.05$ & 1.52 \\
\hline 0.008 & 1.8 & $\ldots$ & $3.88 \pm 0.05$ & $3.62 \pm 0.05$ & 1.39 \\
\hline 0.008 & 1.9 & $\ldots$ & $3.86 \pm 0.04$ & $3.6 \pm 0.03$ & 1.34 \\
\hline 0.009 & 1.0 & $\ldots$ & $3.9 \pm 0.02$ & $3.66 \pm 0.03$ & 1.25 \\
\hline 0.009 & 1.1 & $\ldots$ & $3.96 \pm 0.05$ & $3.7 \pm 0.05$ & 1.48 \\
\hline 0.009 & 1.2 & $\ldots$ & $3.96 \pm 0.04$ & $3.71 \pm 0.05$ & 1.51 \\
\hline 0.009 & 1.3 & $\ldots$ & $3.78 \pm 0.05$ & $3.53 \pm 0.04$ & 2.21 \\
\hline 0.009 & 1.4 & $\ldots$ & $3.91 \pm 0.04$ & $3.65 \pm 0.05$ & 1.47 \\
\hline 0.009 & 1.5 & $\ldots$ & $3.86 \pm 0.04$ & $3.63 \pm 0.05$ & 1.32 \\
\hline 0.009 & 1.6 & $\ldots$ & $3.93 \pm 0.02$ & $3.67 \pm 0.01$ & 1.21 \\
\hline 0.009 & 1.7 & $\ldots$ & $3.84 \pm 0.04$ & $3.59 \pm 0.04$ & 1.3 \\
\hline 0.009 & 1.8 & $\ldots$ & $3.85 \pm 0.04$ & $3.6 \pm 0.03$ & 1.65 \\
\hline 0.009 & 1.9 & $\ldots$ & $3.95 \pm 0.05$ & $3.69 \pm 0.04$ & 1.44 \\
\hline 0.01 & 1.0 & $\ldots$ & $3.9 \pm 0.02$ & $3.65 \pm 0.03$ & 1.71 \\
\hline 0.01 & 1.1 & $\ldots$ & $3.93 \pm 0.04$ & $3.69 \pm 0.03$ & 1.34 \\
\hline 0.01 & 1.2 & $\ldots$ & $3.93 \pm 0.04$ & $3.68 \pm 0.05$ & 1.5 \\
\hline 0.01 & 1.3 & $\ldots$ & $3.83 \pm 0.05$ & $3.58 \pm 0.04$ & 1.74 \\
\hline 0.01 & 1.4 & $\ldots$ & $3.94 \pm 0.05$ & $3.69 \pm 0.05$ & 1.37 \\
\hline 0.01 & 1.5 & $\ldots$ & $3.8 \pm 0.04$ & $3.56 \pm 0.04$ & 1.3 \\
\hline 0.01 & 1.6 & $\ldots$ & $3.88 \pm 0.02$ & $3.63 \pm 0.03$ & 1.26 \\
\hline 0.01 & 1.7 & $\ldots$ & $3.82 \pm 0.05$ & $3.57 \pm 0.04$ & 1.82 \\
\hline 0.01 & 1.8 & $\ldots$ & $3.88 \pm 0.02$ & $3.62 \pm 0.03$ & 1.52 \\
\hline 0.01 & 1.9 & $\ldots$ & $3.82 \pm 0.01$ & $3.56 \pm 0.03$ & 1.49 \\
\hline 0.02 & 1.0 & $\ldots$ & $3.93 \pm 0.04$ & $3.68 \pm 0.05$ & 1.97 \\
\hline 0.02 & 1.1 & $\ldots$ & $3.84 \pm 0.01$ & $3.6 \pm 0.01$ & 1.96 \\
\hline 0.02 & 1.2 & $\ldots$ & $3.88 \pm 0.04$ & $3.63 \pm 0.03$ & 1.2 \\
\hline 0.02 & 1.3 & $\ldots$ & $3.92 \pm 0.04$ & $3.67 \pm 0.03$ & 1.4 \\
\hline 0.02 & 1.4 & $\ldots$ & $3.91 \pm 0.05$ & $3.66 \pm 0.05$ & 1.51 \\
\hline 0.02 & 1.5 & $\ldots$ & $3.75 \pm 0.01$ & $3.5 \pm 0.01$ & 1.72 \\
\hline 0.02 & 1.6 & $\ldots$ & $3.79 \pm 0.04$ & $3.55 \pm 0.03$ & 1.2 \\
\hline 0.02 & 1.7 & $\ldots$ & $3.94 \pm 0.04$ & $3.68 \pm 0.05$ & 1.35 \\
\hline 0.02 & 1.8 & $\ldots$ & $3.93 \pm 0.04$ & $3.67 \pm 0.03$ & 1.31 \\
\hline 0.02 & 1.9 & $\ldots$ & $3.88 \pm 0.04$ & $3.62 \pm 0.03$ & 1.09 \\
\hline 0.03 & 1.0 & $\ldots$ & $3.74 \pm 0.01$ & $3.49 \pm 0.03$ & 1.85 \\
\hline 0.03 & 1.1 & $\ldots$ & $3.88 \pm 0.05$ & $3.63 \pm 0.05$ & 1.61 \\
\hline 0.03 & 1.2 & $\ldots$ & $3.91 \pm 0.02$ & $3.66 \pm 0.03$ & 1.66 \\
\hline 0.03 & 1.3 & $\ldots$ & $3.89 \pm 0.05$ & $3.65 \pm 0.04$ & 1.91 \\
\hline 0.03 & 1.4 & $\ldots$ & $3.89 \pm 0.02$ & $3.63 \pm 0.03$ & 1.55 \\
\hline 0.03 & 1.5 & $\ldots$ & $3.78 \pm 0.01$ & $3.54 \pm 0.03$ & 1.22 \\
\hline 0.03 & 1.6 & $\ldots$ & $3.79 \pm 0.04$ & $3.54 \pm 0.04$ & 1.48 \\
\hline 0.03 & 1.7 & $\ldots$ & $3.82 \pm 0.04$ & $3.57 \pm 0.03$ & 1.52 \\
\hline 0.03 & 1.8 & $\ldots$ & $3.85 \pm 0.02$ & $3.59 \pm 0.01$ & 0.998 \\
\hline 0.03 & 1.9 & $\ldots$ & $3.86 \pm 0.04$ & $3.6 \pm 0.05$ & 1.08 \\
\hline 0.04 & 1.0 & $\ldots$ & $3.91 \pm 0.05$ & $3.66 \pm 0.05$ & 1.53 \\
\hline 0.04 & 1.1 & $\ldots$ & $3.89 \pm 0.04$ & $3.65 \pm 0.05$ & 1.6 \\
\hline 0.04 & 1.2 & $\ldots$ & $3.92 \pm 0.04$ & $3.66 \pm 0.05$ & 1.73 \\
\hline 0.04 & 1.3 & $\ldots$ & $3.8 \pm 0.01$ & $3.56 \pm 0.03$ & 1.68 \\
\hline 0.04 & 1.4 & $\ldots$ & $3.93 \pm 0.04$ & $3.68 \pm 0.03$ & 1.03 \\
\hline 0.04 & 1.5 & $\ldots$ & $3.97 \pm 0.04$ & $3.71 \pm 0.03$ & 1.22 \\
\hline 0.04 & 1.6 & $\ldots$ & $3.86 \pm 0.02$ & $3.61 \pm 0.01$ & 1.39 \\
\hline 0.04 & 1.7 & $\ldots$ & $3.83 \pm 0.01$ & $3.57 \pm 0.03$ & 1.23 \\
\hline 0.04 & 1.8 & $\ldots$ & $3.77 \pm 0.04$ & $3.51 \pm 0.03$ & 1.18 \\
\hline 0.04 & 1.9 & $\ldots$ & $3.81 \pm 0.04$ & $3.54 \pm 0.03$ & 1.32 \\
\hline 0.05 & 1.0 & $\ldots$ & $3.87 \pm 0.04$ & $3.63 \pm 0.05$ & 0.971 \\
\hline 0.05 & 1.1 & $\ldots$ & $3.85 \pm 0.01$ & $3.6 \pm 0.03$ & 1.33 \\
\hline
\end{tabular}

Table 1

(Continued)

\begin{tabular}{|c|c|c|c|c|c|}
\hline$A$ & $\gamma$ & & $\alpha$ & $\beta$ & $\chi^{2} / v$ \\
\hline 0.05 & 1.2 & $\ldots$ & $3.8 \pm 0.04$ & $3.55 \pm 0.03$ & 1.18 \\
\hline 0.05 & 1.3 & $\ldots$ & $3.88 \pm 0.04$ & $3.63 \pm 0.03$ & 1.39 \\
\hline 0.05 & 1.4 & $\ldots$ & $3.9 \pm 0.04$ & $3.64 \pm 0.03$ & 1.29 \\
\hline 0.05 & 1.5 & $\ldots$ & $3.96 \pm 0.04$ & $3.69 \pm 0.05$ & 1.27 \\
\hline 0.05 & 1.6 & $\ldots$ & $3.85 \pm 0.04$ & $3.59 \pm 0.05$ & 1.42 \\
\hline 0.05 & 1.7 & $\ldots$ & $3.89 \pm 0.05$ & $3.62 \pm 0.05$ & 1.19 \\
\hline 0.05 & 1.8 & $\ldots$ & $3.77 \pm 0.04$ & $3.5 \pm 0.04$ & 1.27 \\
\hline 0.05 & 1.9 & $\ldots$ & $3.72 \pm 0.01$ & $3.45 \pm 0.01$ & 1.45 \\
\hline 0.06 & 1.0 & $\ldots$ & $3.88 \pm 0.04$ & $3.62 \pm 0.05$ & 1.36 \\
\hline 0.06 & 1.1 & $\ldots$ & $3.86 \pm 0.04$ & $3.61 \pm 0.03$ & 1.62 \\
\hline 0.06 & 1.2 & $\ldots$ & $3.8 \pm 0.04$ & $3.55 \pm 0.03$ & 1.37 \\
\hline 0.06 & 1.3 & $\ldots$ & $3.91 \pm 0.02$ & $3.66 \pm 0.03$ & 1.72 \\
\hline 0.06 & 1.4 & $\ldots$ & $3.86 \pm 0.04$ & $3.6 \pm 0.03$ & 1.19 \\
\hline 0.06 & 1.5 & $\ldots$ & $3.77 \pm 0.04$ & $3.52 \pm 0.04$ & 1.6 \\
\hline 0.06 & 1.6 & $\ldots$ & $3.88 \pm 0.04$ & $3.61 \pm 0.03$ & 1.31 \\
\hline 0.06 & 1.7 & $\ldots$ & $3.73 \pm 0.04$ & $3.46 \pm 0.03$ & 1.52 \\
\hline 0.06 & 1.8 & $\ldots$ & $3.76 \pm 0.01$ & $3.49 \pm 0.03$ & 1.14 \\
\hline 0.06 & 1.9 & $\ldots$ & $3.82 \pm 0.04$ & $3.55 \pm 0.05$ & 1.29 \\
\hline 0.07 & 1.0 & $\ldots$ & $3.85 \pm 0.04$ & $3.61 \pm 0.03$ & 1.58 \\
\hline 0.07 & 1.1 & $\ldots$ & $3.74 \pm 0.01$ & $3.5 \pm 0.01$ & 1.28 \\
\hline 0.07 & 1.2 & $\ldots$ & $3.87 \pm 0.02$ & $3.62 \pm 0.03$ & 1.67 \\
\hline 0.07 & 1.3 & $\ldots$ & $3.87 \pm 0.04$ & $3.61 \pm 0.03$ & 1.47 \\
\hline 0.07 & 1.4 & $\ldots$ & $3.86 \pm 0.05$ & $3.6 \pm 0.05$ & 1.8 \\
\hline 0.07 & 1.5 & $\ldots$ & $3.91 \pm 0.05$ & $3.65 \pm 0.05$ & 1.34 \\
\hline 0.07 & 1.6 & $\ldots$ & $3.78 \pm 0.04$ & $3.53 \pm 0.03$ & 1.36 \\
\hline 0.07 & 1.7 & $\ldots$ & $3.8 \pm 0.04$ & $3.54 \pm 0.04$ & 1.49 \\
\hline 0.07 & 1.8 & $\ldots$ & $3.78 \pm 0.01$ & $3.5 \pm 0.03$ & 1.72 \\
\hline 0.07 & 1.9 & $\ldots$ & $3.87 \pm 0.02$ & $3.59 \pm 0.01$ & 1.01 \\
\hline 0.08 & 1.0 & $\ldots$ & $3.86 \pm 0.04$ & $3.6 \pm 0.03$ & 1.42 \\
\hline 0.08 & 1.1 & $\ldots$ & $3.9 \pm 0.05$ & $3.64 \pm 0.04$ & 1.28 \\
\hline 0.08 & 1.2 & $\ldots$ & $3.88 \pm 0.04$ & $3.62 \pm 0.03$ & 1.03 \\
\hline 0.08 & 1.3 & $\ldots$ & $3.89 \pm 0.05$ & $3.63 \pm 0.05$ & 1.47 \\
\hline 0.08 & 1.4 & $\ldots$ & $3.79 \pm 0.04$ & $3.54 \pm 0.04$ & 1.53 \\
\hline 0.08 & 1.5 & $\ldots$ & $3.79 \pm 0.05$ & $3.53 \pm 0.04$ & 1.94 \\
\hline 0.08 & 1.6 & $\ldots$ & $3.9 \pm 0.04$ & $3.64 \pm 0.03$ & 1.29 \\
\hline 0.08 & 1.7 & $\ldots$ & $3.76 \pm 0.04$ & $3.5 \pm 0.04$ & 1.54 \\
\hline 0.08 & 1.8 & $\ldots$ & $3.7 \pm 0.04$ & $3.42 \pm 0.04$ & 1.98 \\
\hline 0.08 & 1.9 & $\ldots$ & $3.92 \pm 0.05$ & $3.63 \pm 0.04$ & 1.15 \\
\hline 0.09 & 1.0 & $\ldots$ & $3.87 \pm 0.02$ & $3.61 \pm 0.03$ & 1.34 \\
\hline 0.09 & 1.1 & $\ldots$ & $3.92 \pm 0.05$ & $3.67 \pm 0.05$ & 1.44 \\
\hline 0.09 & 1.2 & $\ldots$ & $3.97 \pm 0.04$ & $3.71 \pm 0.05$ & 0.92 \\
\hline 0.09 & 1.3 & $\ldots$ & $3.84 \pm 0.05$ & $3.59 \pm 0.05$ & 1.63 \\
\hline 0.09 & 1.4 & $\ldots$ & $3.94 \pm 0.04$ & $3.67 \pm 0.05$ & 1.87 \\
\hline 0.09 & 1.5 & $\ldots$ & $3.75 \pm 0.04$ & $3.49 \pm 0.04$ & 1.57 \\
\hline 0.09 & 1.6 & $\ldots$ & $3.95 \pm 0.04$ & $3.67 \pm 0.03$ & 1.44 \\
\hline 0.09 & 1.7 & $\ldots$ & $3.79 \pm 0.04$ & $3.53 \pm 0.05$ & 1.38 \\
\hline 0.09 & 1.8 & $\ldots$ & $3.92 \pm 0.02$ & $3.63 \pm 0.03$ & 1.41 \\
\hline 0.09 & 1.9 & $\ldots$ & $3.86 \pm 0.02$ & $3.58 \pm 0.03$ & 0.993 \\
\hline 0.1 & 1.0 & $\ldots$ & $3.87 \pm 0.04$ & $3.62 \pm 0.05$ & 1.76 \\
\hline 0.1 & 1.1 & $\ldots$ & $3.91 \pm 0.02$ & $3.66 \pm 0.03$ & 1.75 \\
\hline 0.1 & 1.2 & $\ldots$ & $3.93 \pm 0.04$ & $3.69 \pm 0.05$ & 1.42 \\
\hline 0.1 & 1.3 & $\ldots$ & $3.85 \pm 0.04$ & $3.6 \pm 0.05$ & 1.18 \\
\hline 0.1 & 1.4 & $\ldots$ & $3.86 \pm 0.04$ & $3.6 \pm 0.03$ & 1.47 \\
\hline 0.1 & 1.5 & $\ldots$ & $3.88 \pm 0.02$ & $3.62 \pm 0.03$ & 1.25 \\
\hline 0.1 & 1.6 & $\ldots$ & $3.85 \pm 0.04$ & $3.57 \pm 0.03$ & 1.47 \\
\hline 0.1 & 1.7 & $\ldots$ & $3.8 \pm 0.04$ & $3.53 \pm 0.04$ & 1.68 \\
\hline 0.1 & 1.8 & $\ldots$ & $3.74 \pm 0.04$ & $3.46 \pm 0.03$ & 1.38 \\
\hline 0.1 & 1.9 & $\ldots$ & $3.76 \pm 0.05$ & $3.49 \pm 0.04$ & 1.55 \\
\hline
\end{tabular}

Poisson particular case. We generate simulated fields spanning a wide range of the parameter space $(A, \gamma)$ around the measured values reported in the literature. These simulated fields are used to characterize the VT cell areas distribution.

Although aimed at application in our cluster finder algorithm, this study allows us to investigate the connection between this 


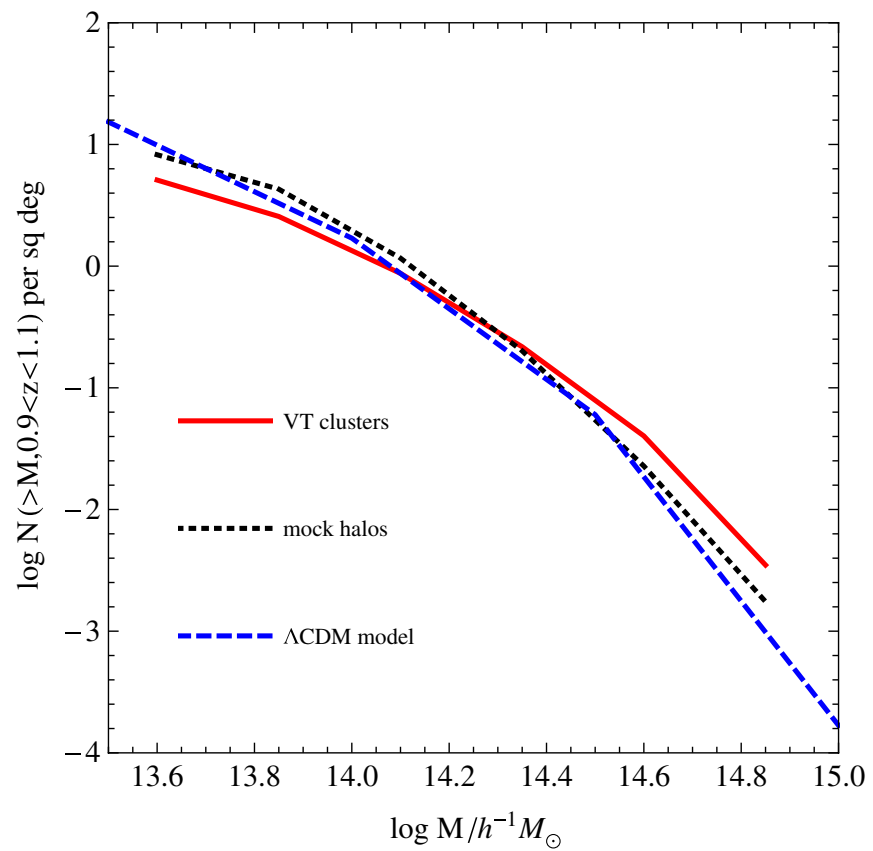

Figure 4. Cumulative cluster abundance as a function of mass in the redshift range $0.9<z<1.1$. The black (dotted) line shows the counts in the truth table; the red solid (solid) line shows the results of the VT catalog, taking $\sigma_{z}=0.015$; the blue (dashed) line shows the values predicted for a $\Lambda$ CDM cosmology.

(A color version of this figure is available in the online journal.)

VT property and the statistical process of the generator set of points. This topic has been extensively discussed (see Okabe 2000 for a review). For the Poisson case, simulations have been used to support the so-called Kiang's conjecture that the distribution of standardized cell sizes (size/mean size) in $n$-dimensional space is given by

$$
p(x)=\frac{\beta^{\alpha}}{\Gamma(\alpha)} x^{\alpha-1} \exp ^{-\beta x}
$$

with $\alpha=\beta=2 n$. This has been rigorously shown for $n=1$ and studied in simulations up to $n=3$. Here we extend this conjecture to the case where the two-point correlation function of the field is given by a power law. We focus on $n=2$. Our results indicate that Equation (A2) still holds, but the parameters $\alpha$ and $\beta$ are modified. The relation $\alpha=0.26+\beta$ is found to be valid within the parameters space explored.

In the following sections, we describe the simulations and the modeling of the area distribution. We discuss our results in comparison to the well-studied Poisson case and provide the relevant quantities in Table 1.

\section{A.1. Point Field Simulation}

To generate the simulated fields with two-point correlation function given by Equation (A1), we implement the simulated annealing method as proposed by Rintoul \& Torquato (1997). This method is generally used to find the state of minimum "energy" of a given system by sampling the different states weighted by the probability of occurrence of that state. Here we take Equation (A1) as our "reference" state, and the state of the "system" is denoted as $w_{s}(\theta)$. We consider logarithmic bins in $\theta$, and define the energy of the system as

$$
E=\sum_{i}\left(w_{s}\left(\theta_{i}\right)-w\left(\theta_{i}\right)\right)^{2}
$$

where the sum is over all bins. We use 10 bins in the interval $0.01<\theta_{i}<2$. This definition of energy is convenient because it ensures that $E$ decreases when the difference between any two bins decreases.

The initial state is a Poisson state. To evolve the system toward $w(\theta)$, we chose a particle and move it to a random position in the field. We compute the energy $E^{\prime}$ of this new configuration and obtain $\Delta E=E^{\prime}-E$. The move is accepted with probability

$$
p(\Delta E)= \begin{cases}1 & \Delta E \leqslant 0 \\ \exp (-\Delta E / k T) & \Delta E>0\end{cases}
$$

where $k T$ is the "temperature" of the system. This is chosen to allow the system to evolve as quickly as possible to the minimum state, without getting trapped in local minima. The initial temperature is set to 1 . We attempt to move all the particles sequentially and, after a complete round over all the $N$ particles of the system, its temperature is cooled by a factor of two. The system converges about $30 \%$ faster with this cooling schedule.

In Figure 5 we show one example, where $A=0.005$ and $\gamma=1.7$. This combination of parameters corresponds to typical values measured, for instance, on SDSS data up to magnitude
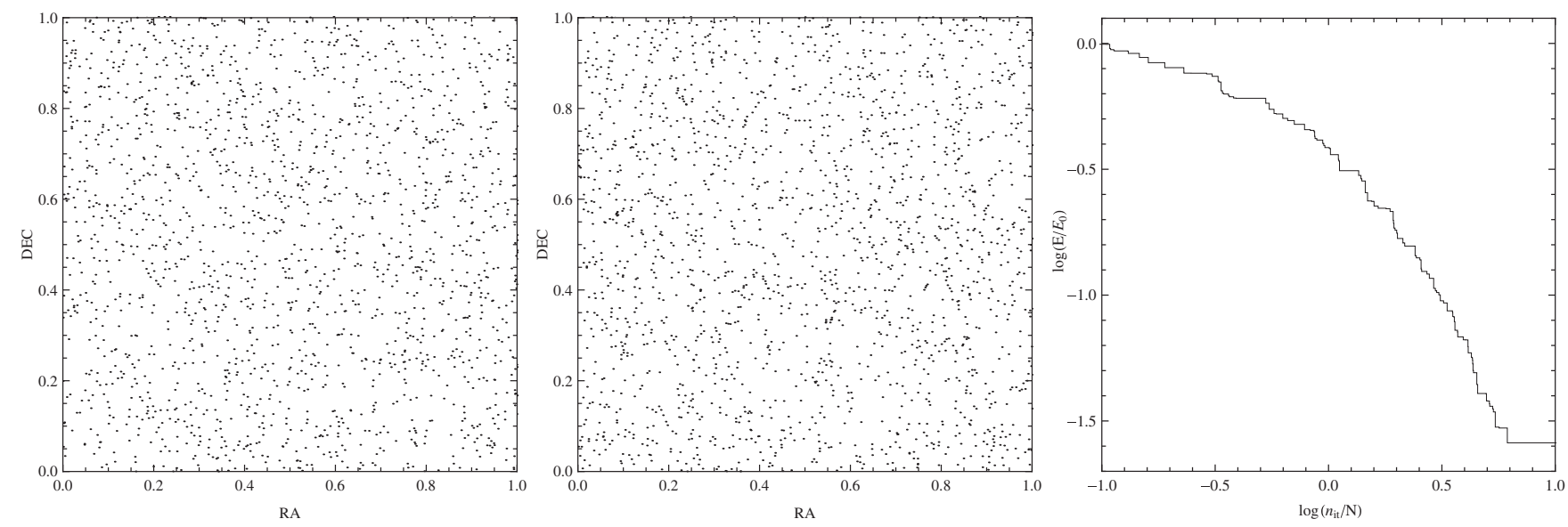

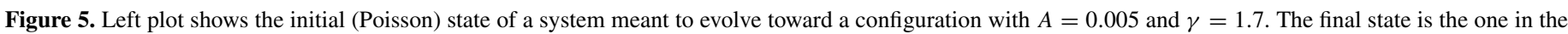

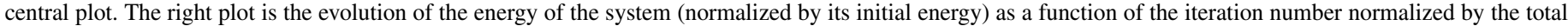

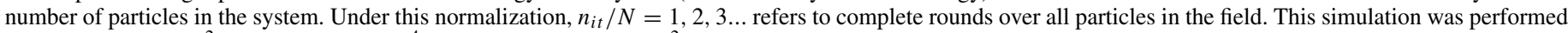
in a box of $3 \times 3 \mathrm{deg}^{2}$ containing $1.6 \times 10^{4}$ particles. Just a $1 \times 1 \mathrm{deg}^{2}$ portion of the field is shown. 

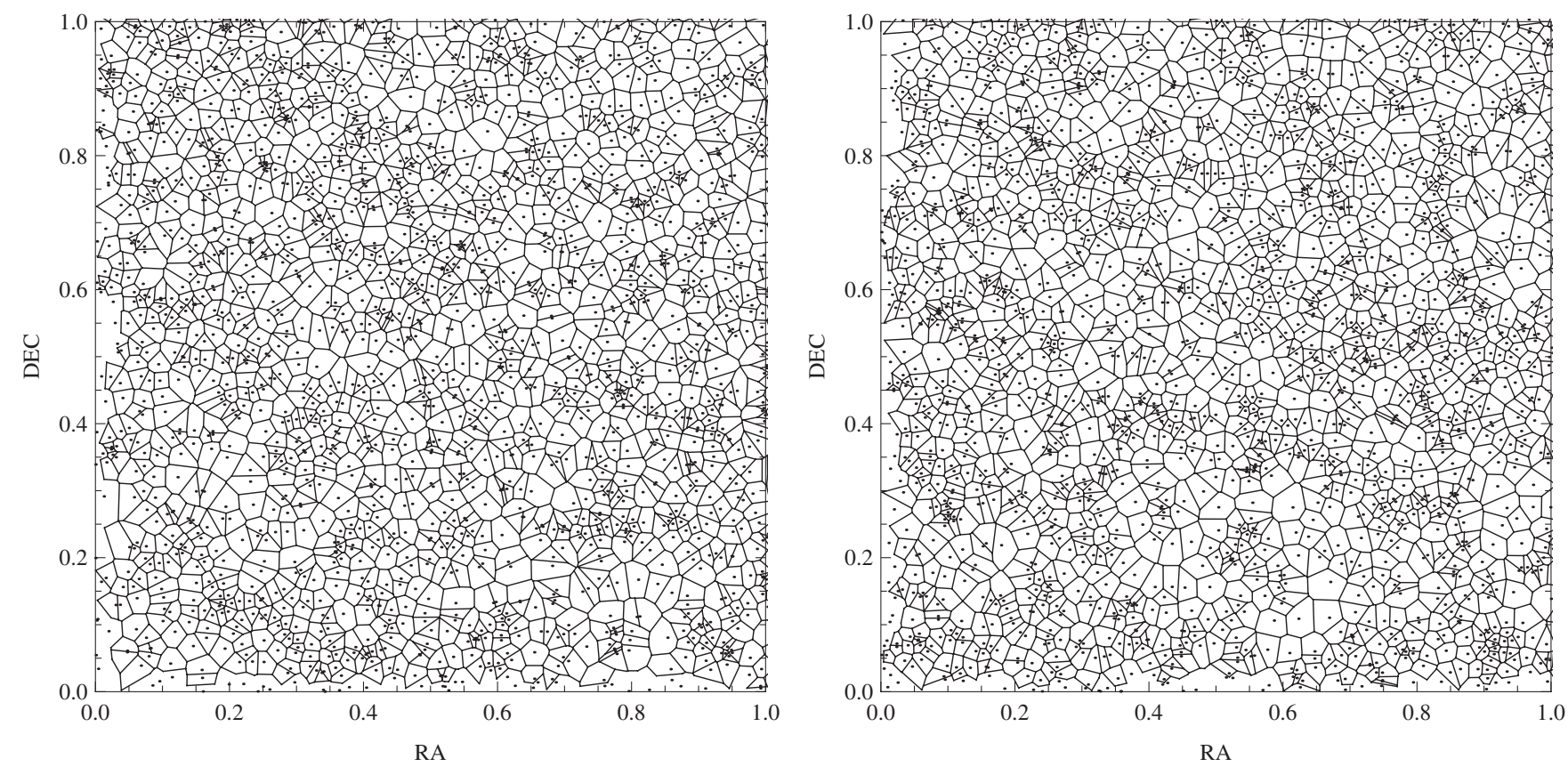

Figure 6. Voronoi diagram corresponding to the two fields shown in Figure 5. The initial and final states are on the left and right panels, respectively.
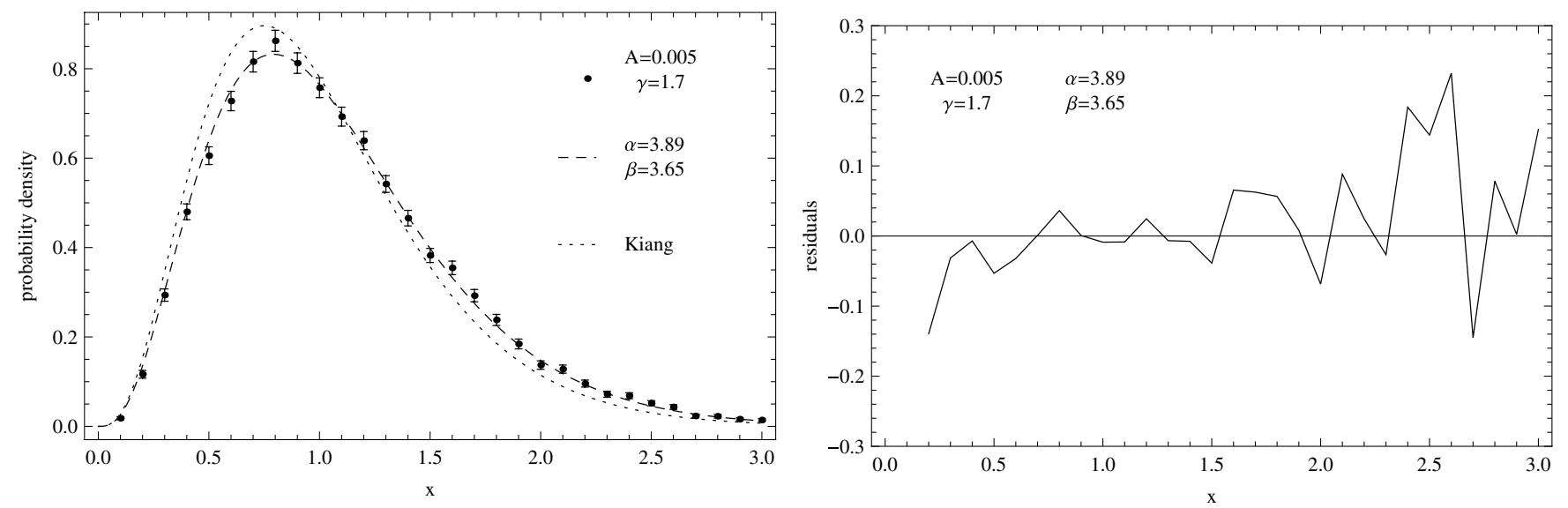

Figure 7. Left: best-fit model for the distribution of normalized VT cell areas featured in Figure 6. The curve for the Poisson case is also shown for comparison (dashed line). Right: fractional residuals of the fit.
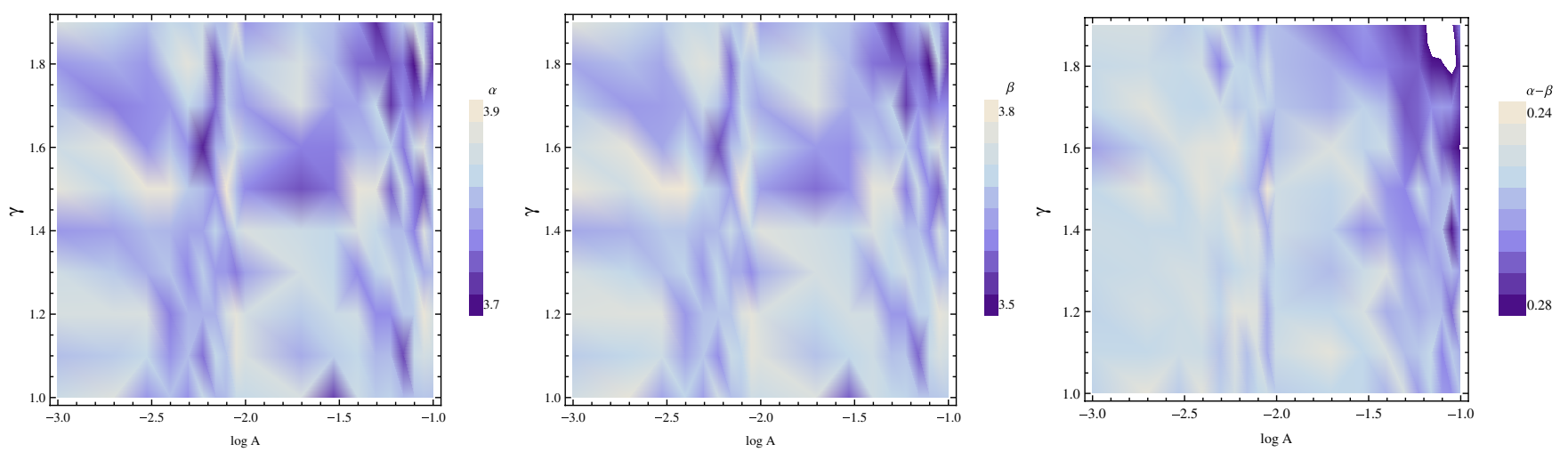

Figure 8. Density maps showing the results of the fit in the parameter space investigated. There is a noticeable correlation between the two leftmost maps. The difference between these two maps is shown in the right.

(A color version of this figure is available in the online journal.) 
limit $r^{\prime}=21.5$ (Connolly et al. 2002). The initial system is on the left, the field in the middle is the final state, after 10 rounds over all particles. The plot on the right shows the evolution of the energy of the system. The difference between the initial and final states is not noticeable by eye and a statistical method must be used to actually measure the two-point correlation function and compute $\Delta E$ at each iteration. We use a fast Fourier transform code (Szapudi et al. 2005) to accomplish this. Using this method we have generated 190 fields of $3 \times 3 \mathrm{deg}^{2}$ and $1.6 \times 10^{4}$ particles.

\section{A.2. Gamma Model for the VT Cell Distribution}

We apply the VT code on each of the simulated fields, obtain the distribution of cell normalized cell areas, and find the best-fit Gamma model (Equation (A2)). Figure 6 shows as an example the VT diagram for the same system featured above. The left and right diagrams correspond to the initial and final states of the system, respectively.

The result of the fit is shown in Figure 7, again for the case $A=0.005$ and $\gamma=1.7$. For comparison we show as well the traditional Kiang formula (dashed line). The results are $\alpha=3.89 \pm 0.04$ and $\beta=3.65 \pm 0.05$. Kiang's formula is more than $5 \sigma$ away from the best fit.

The results for the ensemble of simulated fields studied are shown in Figure 8. The values of $\alpha$ and $\beta$ fall in the range $3.5<\alpha<3.9$ and $3.5<\beta<3.8$. The mean error in both is 0.04 . There is a noticeable correlation between these two parameters. The difference $\alpha-\beta$ is shown to be $0.26 \pm 0.02$ all over the parameter space explored. The model parameters for the values of $A$ and $\gamma$ considered are presented in Table 1.

\section{REFERENCES}

Abell, G. O. 1958, ApJS, 3, 211

Abell, G. O., Corwin, Jr. H. G., \& Olowin, R. P. 1989, ApJS, 70, 1

Annis, J., et al. 1999, BAAS, 31, 1391

Bahcall, N. A. 1986, ApJ, 302, L41

Bahcall, N. A., \& Soneira, R. M. 1983, ApJ, 270, 20

Bahcall, N. A., et al. 2003, ApJS, 148, 243

Barkhouse, W. A., et al. 2006, ApJ, 645, 955

Bartelmann, M., \& White, S. D. M. 2002, A\&A, 388, 732

Berlind, A. A., et al. 2006, ApJS, 167, 1

Blanton, M. R., et al. 2003, ApJ, 592, 819

Busha, M., \& Wechsler, R. 2008, in Proc. 43rd Rencontres de Moriond, ed. J. Dumarchez, Y. Giraud-Heraud, \& J. Tran Thanh Van (Hà Nôi: Thê Giói Publishers), http://moriond.in2p3.fr/J08/proceedings/busha.pdf

Connolly, A. J., et al. 2002, ApJ, 579, 42

Cooper, M. C., et al. 2007, MNRAS, 376, 1445

Dalton, G. B., Maddox, S. J., Sutherland, W. J., \& Efstathiou, G. 1997, MNRAS, 289, 263

Davis, M., \& Peebles, P. J. E. 1983, ApJ, 267, 465

Diehl, S., \& Statler, T. S. 2006, MNRAS, 368, 497

Doroshkevich, A. G., Gottlober, S., \& Madsen, S. 1997, A\&AS, 123, 495

Ebeling, H., \& Wiedenmann, G. 1993, Phys. Rev. E, 47, 704

Eke, V. R., et al. 2004, MNRAS, 348, 866

El-Ad, H., Piran, T., \& da Costa, L. N. 1996, ApJ, 462, L13
Evrard, A. E., et al. 2002, ApJ, 573, 7

Gal, R. R., de Carvalho, R. R., Brunner, R., Odewahn, S. C., \& Djorgovski, S. G. 2000, AJ, 120, 540

Gal, R. R., de Carvalho, R. R., Lopes, P. A. A., Djorgovski, S. G., Brunner, R. J., Mahabal, A., \& Odewahn, S. C. 2003, AJ, 125, 2064

Gal, R. R., Lopes, P. A. A., de Carvalho, R. R., Kohl-Moreira, J. L., Capelato, H. V., \& Djorgovski, S. G. 2009, AJ, 137, 2981

Gerdes, D. W., Sypniewski, A. J., McKay, T. A., Hao, J., Weis, M. R., Wechsler, R. H., \& Busha, M. T. 2010, ApJ, 715, 823

Gerke, B. F., et al. 2005, ApJ, 625, 6

Gladders, M. D., \& Yee, H. K. C. 2005, ApJS, 157, 1

Goto, T., et al. 2002, AJ, 123, 1807

Haiman, Z., Mohr, J. J., \& Holder, G. P. 2001, ApJ, 553, 545

Hansen, S. M., McKay, T. A., Wechsler, R. H., Annis, J., Sheldon, E. S., \& Kimball, A. 2005, ApJ, 633, 122

Hao, J. 2009, PhD thesis, Univ. Michigan

Holder, G., Haiman, Z., \& Mohr, J. J. 2001, ApJ, 560, L111

Hu, W. 2003, Phys. Rev. D, 67, 081304

Huchra, J. P., \& Geller, M. J. 1982, ApJ, 257, 423

Ikeuchi, S., \& Turner, E. L. 1991, MNRAS, 250, 519

Kiang, T. 1966, Z. Astrophys., 64, 433

Kim, R. S. J., et al. 2002, AJ, 123, 20

Koester, B. P., et al. 2007a, ApJ, 660, 239

Koester, B. P., et al. 2007b, ApJ, 660, 221

La Barbera, F., de Carvalho, R. R., Kohl-Moreira, J. L., Gal, R. R., SoaresSantos, M., Capaccioli, M., Santos, R., \& Santánna, N. 2008, PASP, 120, 681

Levine, E. S., Schulz, A. E., \& White, M. 2002, ApJ, 577, 569

Lobo, C., Iovino, A., Lazzati, D., \& Chincarini, G. 2000, A\&A, 360, 896

Lopes, P. A. A., de Carvalho, R. R., Gal, R. R., Djorgovski, S. G., Odewahn, S. C., Mahabal, A. A., \& Brunner, R. J. 2004, AJ, 128, 1017

Lumsden, S. L., Nichol, R. C., Collins, C. A., \& Guzzo, L. 1992, MNRAS, 258, 1

Miller, C. J., et al. 2005, AJ, 130, 968

Okabe, A. 2000, Spatial Tessellations: Concepts and Applications of Voronoi Diagrams (2nd ed.; New York: Wiley)

Postman, M., Lubin, L. M., Gunn, J. E., Oke, J. B., Hoessel, J. G., Schneider, D. P., \& Christensen, J. A. 1996, AJ, 111, 615

Ramella, M., Boschin, W., Fadda, D., \& Nonino, M. 2001, A\&A, 368, 776

Rintoul, M. D., \& Torquato, S. 1997, J. Colloid Interface Sci., 186, 467

Rozo, E., et al. 2007, arXiv:astro-ph/0703571

Rozo, E., et al. 2009, ApJ, 703, 601

Rozo, E., et al. 2010, ApJ, 708, 645

Shewchuk, J. R. 1996, in Lecture Notes in Computer Science, Vol. 1148, Applied Computational Geometry: Towards Geometric Engineering, ed. M. C. Lin \& D. Manocha (Berlin: Springer), 203

Szapudi, I., Pan, J., Prunet, S., \& Budavári, T. 2005, ApJ, 631, L1

van Breukelen, C., \& Clewley, L. 2009, MNRAS, 395, 1845

van Breukelen, C., et al. 2006, MNRAS, 373, L26

van de Weygaert, R. 1994, A\&A, 283, 361

van de Weygaert, R., \& Icke, V. 1989, A\&A, 213, 1

Vikhlinin, A., et al. 2009, ApJ, 692, 1060

Wechsler, R. H. 2004, in Carnegie Obs. Astrophys. Ser. Vol. 3, Clusters of Galaxies: Probes of Cosmological Structure and Galaxy Evolution, ed. J. S. Mulchaey, A. Dressler, \& A. Oemler (Pasadena, CA: Carnegie Obs.), 53

White, M., \& Kochanek, C. S. 2002, ApJ, 574, 24

Zaninetti, L. 1995, A\&AS, 109, 71

Zaritsky, D., Gonzalez, A. H., Nelson, A. E., \& Dalcanton, J. J. 2002, in ASP Conf. Ser. 257, AMiBA 2001: High-Z Clusters, Missing Baryons, and CMB Polarization, ed. L.-W. Chen et al. (San Francisco, CA: ASP), 133

Zaritsky, D., Nelson, A. E., Dalcanton, J. J., \& Gonzalez, A. H. 1997, ApJ, 480, L91 